\title{
Asymmetric Synthesis of Functionalized Dihydro- and Tetrahydropyrans via an Organocatalytic Domino Michael-Hemiacetalization Reaction
}

\author{
Gregor Urbanietz, Iuliana Atodiresei, Dieter Enders* \\ Institute of Organic Chemistry, RWTH Aachen University, Landoltweg 1, 52074 Aachen, Germany \\ Fax +49(241)8092127; E-mail: enders@rwth-aachen.de \\ Received: 22.01.2014; Accepted: 24.01.2014
}

License terms: (c) (9)

\begin{abstract}
Starting from $\alpha$-hydroxymethyl nitroalkenes and various 1,3-dicarbonyl compounds, a one-pot organocatalyzed diastereoand enantioselective synthesis of polyfunctionalized dihydro- and tetrahydropyran derivatives via a domino Michael-hemiacetalization sequence is reported. The title compounds bearing a variety of functional groups can be synthesized in this way in good yields (59$91 \%)$ and with moderate to excellent diastereoselectivities (26$98 \%$ de) and enantioselectivities (71-99\% ee).
\end{abstract}

Key words: organocatalysis, domino reaction, one-pot reaction, tetrahydropyrans, hydrogen bonding

Great progress has been made in the organocatalytic asymmetric synthesis of chiral molecules in a single operation via the concept of domino reactions. ${ }^{1}$ So far the development of methods for the control of multiple stereocenters was mainly dominated by amino activation modes via Lewis base catalysis or hydrogen bonding activation as another elegant option. ${ }^{2}$ Owing to the importance of chiral tetrahydropyran structures, which are characteristic structural features of many natural products, these heterocycles have attracted considerable attention in recent years and many methods have been developed for the preparation of this framework. ${ }^{3,4}$ Recently we and others reported that chromanols $\mathbf{6}$ can be efficiently synthesized through a Michael addition of 1,3-dicarbonyl compounds $\mathbf{1}$ to nitrovinylphenol $\mathbf{5}$ and subsequent intramolecular hemiacetalization. ${ }^{5}$ The hydroxyl group participates in this process through carbonyl addition and makes this sequence a powerful tool among the existing annulation methods (Scheme 1, a).

We now would like to report an efficient organocatalytic asymmetric synthesis of functionalized tetrahydropyranols 3 by hydrogen bonding activation of $\alpha$-hydroxymethyl nitroalkenes 2 as Michael acceptors via a domino Michael-hemiacetalization reaction followed by dehydration to form dihydropyrans 4 (Scheme $1, \mathrm{~b}) .^{4 \mathrm{~b}, \mathrm{~h}-\mathrm{j}, 6}$

We started our studies on the domino Michael-hemiacetalization reactions by screening the organocatalysts $\mathbf{A}$ E $(10 \mathrm{~mol} \%)$ for the Michael reaction of $(E)$-2-nitro-3phenylprop-2-en-1-ol (2a) ${ }^{7}$ with 1 equivalent of methyl 3oxobutanoate (1a) in toluene (Table 1, entries 1-5). The intermediate tetrahydropyranol 3a was directly dehydrated to the 3,4-dihydro-2 $H$-pyran 4a in a one-pot procedure

SYNTHESIS 2014, 46, 1261-1269

Advanced online publication: 18.02.2014

DOI: 10.1055/s-0033-1340826; Art ID: SS-2014-Z0060-OP

(C) Georg Thieme Verlag Stuttgart · New York

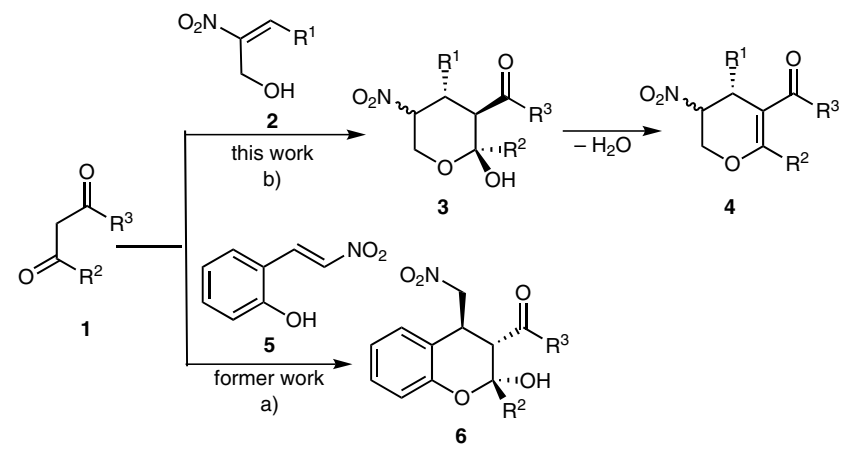

Scheme 1 Domino Michael-hemiacetalization reactions towards chromanols $\mathbf{6}$ or tetrahydropyranols $\mathbf{3}$ and dihydropyrans $\mathbf{4}$

(see Table 1). It turned out that $20 \mathrm{~mol} \%$ PTSA in toluene at $100{ }^{\circ} \mathrm{C}$ for one hour is the best reaction condition for the dehydration process, which occurred in good yields and with no competing ring opening and lactonization. With this reaction procedure the diastereo- and enantiomeric excess of $4 \mathbf{a}$ were determined by HPLC. While the thiourea derived catalysts $\mathbf{A}, \mathbf{B}$, and $\mathbf{D}$ showed moderate to good diastereo- and enantioselectivities, the square amide derived catalyst $\mathbf{E}^{8}$ led to the best results (Table 1, entry 5). The monodentate carbamate derived catalyst $\mathbf{C}^{9}$ gave the poorest enantioselectivity but had not much of an impact on the diastereoselectivity (Table 1, entry 3 ). Solvents like toluene or THF also have only a slight impact on the diastereoselectivity (Table 1, entries 5,6) while dichloromethane showed the best diastereomeric excess value (Table 1, entry 7). With catalyst $\mathbf{E}$ we tried to improve the stereoselectivity as well as the yield of the reaction by decreasing the temperature. The domino Michaelhemiacetalization reaction proceeds at $5{ }^{\circ} \mathrm{C}$ as well as at $-25^{\circ} \mathrm{C}$, but has only a slight effect with regard to selectivity and longer reaction times were necessary (Table 1, entries 8,9$)$. Next we decided to change the catalyst loading from $10 \mathrm{~mol} \%$ over $5 \mathrm{~mol} \%$ to $1 \mathrm{~mol} \%$ at ambient temperature (Table 1 , entries 10,11 ) and this had only an impact on the reaction time and not on the yield or selectivity. In summary, the diastereomeric excess remained in the range of $61-71 \%$ in favor of the trans-isomer and could not be improved by catalysts, loading, temperature, or solvents.

In order to determine the scope of the organocatalytic onepot reaction a number of $(E)$ - 3-aryl-2-nitroprop-2-en-1ols 2 were reacted with 1 equivalent of various 1,3 dicarbonyl compounds 1 in the presence of $10 \mathrm{~mol} \%$ of the catalyst $\mathbf{E}$ at ambient temperature in dichloromethane. The 
dihydropyrans $\mathbf{4 a}-\mathbf{h}$ were obtained in good yields and stereoselectivities (Table 2). In the cases of different $\beta$-keto esters $\mathbf{1}$, a high increase in stereoselectivity was observed by using the phenyl substituted $\beta$-keto ester 1c (Table 2, $\mathbf{4 c})$. In contrast to acetylacetone (Table $2,4 \mathbf{d}$ ) a reaction with malonates could not be observed. While using various hydroxymethyl-substituted nitroalkenes 2 good yields (81-86\%) and enantioselectivities (78-94\% ee) could be achieved. Electronic factors had a great influence on the diastereoselectivity. Both the neutral and the electron-donating group substituted nitrostyrenes 2 led to the expected products with diastereoselectivities of $26-76 \%$ de (Table 2, 4e-h). The lowest diastereoselectivity was ob- tained by using the thienyl-substituted hydroxymethylene nitroolefin (Table 2, product $\mathbf{4 g}$ ), nevertheless the enantioselectivity was excellent $(90 / 94 \%$ ee). In all cases we were unable to separate the diastereomers by column chromatography. Therefore, this protocol was used for the synthesis of the corresponding lactols (Table 3, products 3a-h). While the enantioselectivity of the tetrahydropyranols 3a-h could not be measured by HPLC, the major trans-3a-h isomers were separated from the minor cis3a-h isomers by column chromatography. While with four adjacent stereogenic centers eight diastereomers of tetrahydropyran derivatives were possible, only two diastereomers could be isolated.

Table 1 Catalyst Screening for the One-Pot Domino Michael-Hemiacetalization and Dehydration Sequence to from 4a

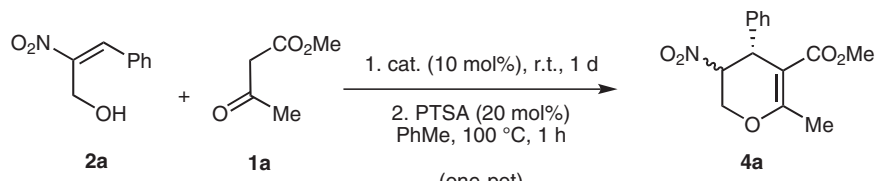<smiles>CN(C)[C@H]1CCCC[C@H]1NC(=S)Nc1cc(C(F)(F)F)cc(C(F)(F)F)c1</smiles>

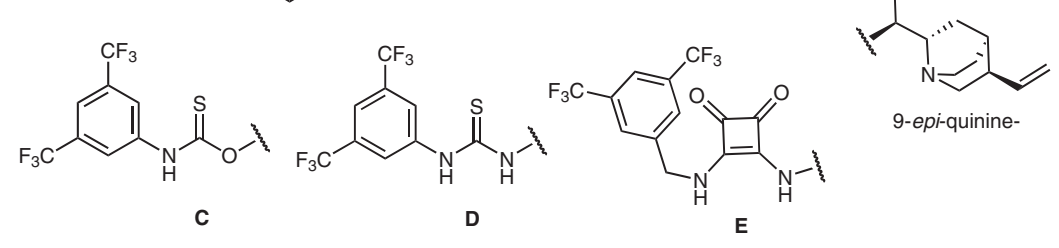

\begin{tabular}{|c|c|c|c|c|c|}
\hline Entry $^{\mathrm{a}}$ & Catalyst & Solvent & Yield $(\%)^{b}$ & de $(\%)^{\mathrm{c}}$ & ee $(\%)^{c, d}$ \\
\hline 1 & $\mathbf{A}$ & toluene & 86 & 66 & $75(57)$ \\
\hline 2 & B & toluene & 83 & 58 & $56(11)^{\mathrm{e}}$ \\
\hline 3 & C & toluene & 72 & 68 & $27(27)$ \\
\hline 4 & D & toluene & 74 & 62 & $51(28)$ \\
\hline 5 & $\mathbf{E}$ & toluene & 81 & 67 & $87(61)$ \\
\hline 6 & $\mathbf{E}$ & THF & 79 & 61 & $86(75)$ \\
\hline 7 & $\mathbf{E}$ & $\mathrm{CH}_{2} \mathrm{Cl}_{2}$ & 80 & 71 & $95(71)$ \\
\hline $8^{f}$ & $\mathbf{E}$ & $\mathrm{CH}_{2} \mathrm{Cl}_{2}$ & 74 & 68 & $91(71)$ \\
\hline $9^{g}$ & $\mathbf{E}$ & $\mathrm{CH}_{2} \mathrm{Cl}_{2}$ & 80 & 69 & $91(73)$ \\
\hline $10^{\mathrm{h}}$ & $\mathbf{E}$ & $\mathrm{CH}_{2} \mathrm{Cl}_{2}$ & 82 & 65 & $88(80)$ \\
\hline $11^{\mathrm{i}}$ & $\mathbf{E}$ & $\mathrm{CH}_{2} \mathrm{Cl}_{2}$ & 88 & 68 & 91 (69) \\
\hline
\end{tabular}

a All reactions were performed on a $0.5 \mathrm{mmol}$ scale.

b Yield of isolated product $\mathbf{4 a}$.

${ }^{c}$ Determined by HPLC analysis on a chiral stationary phase.

$\mathrm{d}$ The enantiomeric excess of the minor diastereomer is given in parentheses.

e ent-4a was obtained.

${ }^{\mathrm{f}}$ Performed with $10 \mathrm{~mol} \%$ catalyst loading at $5{ }^{\circ} \mathrm{C}$ for $2 \mathrm{~d}$.

g Performed with $10 \mathrm{~mol} \%$ catalyst loading at $-25^{\circ} \mathrm{C}$ for $3 \mathrm{~d}$.

${ }^{\mathrm{h}}$ Performed with $5 \mathrm{~mol} \%$ catalyst loading at r.t. for $7 \mathrm{~d}$.

${ }^{\mathrm{i}}$ Performed with $1 \mathrm{~mol} \%$ catalyst loading at r.t. for $14 \mathrm{~d}$. 
Table 2 Scope of the Asymmetric Synthesis of the Dihydropyran Derivates 4 via a One-Pot Domino Michael-Hemiacetalization and Dehydration Reaction

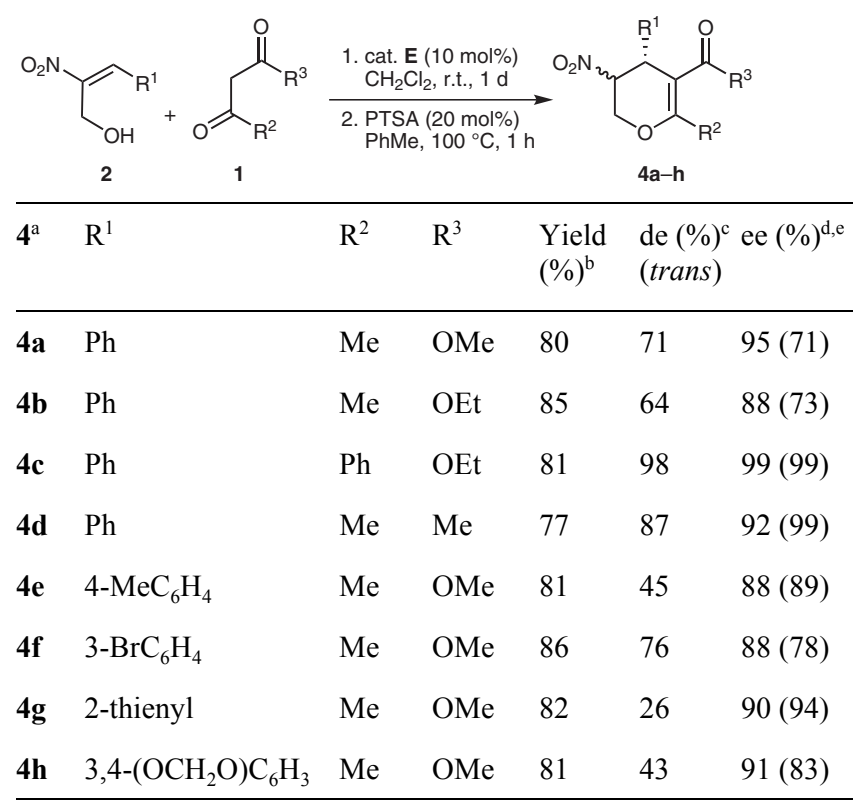

${ }^{\text {a }}$ All reactions were performed on a $1.0 \mathrm{mmol}$ scale.

${ }^{\mathrm{b}}$ Yield of isolated product.

${ }^{\mathrm{c}}$ Determined by HPLC analysis.

${ }^{\mathrm{d}}$ Determined by HPLC analysis on a chiral stationary phase.

e The enantiomeric excess of the minor cis-diastereomer is given in parentheses.

Table 3 Scope of the Asymmetric Synthesis of the Tetrahydropyranols 3 via Domino Michael-Hemiacetalization Reaction

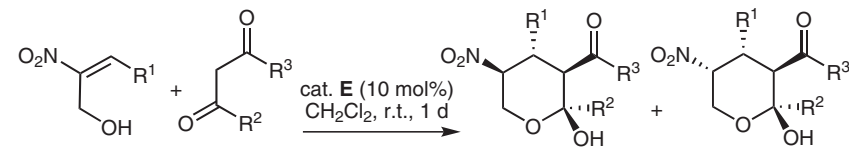

2

trans-3a-h

cis-3a-h

\begin{tabular}{llllll}
\hline 3 $^{\mathrm{a}}$ & $\mathrm{R}^{1}$ & $\mathrm{R}^{2}$ & $\mathrm{R}^{3}$ & $\begin{array}{l}\text { Yield }(\%)^{\mathrm{b}} \\
\text { trans } \mathbf{3}\end{array}$ & $\begin{array}{l}\text { Yield }(\%)^{\mathrm{b}} \\
\text { cis-3 }\end{array}$ \\
\hline 3a & $\mathrm{Ph}$ & $\mathrm{Me}$ & $\mathrm{OMe}$ & 80 & 14 \\
3b & $\mathrm{Ph}$ & $\mathrm{Me}$ & $\mathrm{OEt}$ & 77 & 18 \\
3c & $\mathrm{Ph}$ & $\mathrm{Ph}$ & $\mathrm{OEt}$ & 91 & 2 \\
3d & $\mathrm{Ph}$ & $\mathrm{Me}$ & $\mathrm{Me}$ & 80 & 6 \\
3e & $4-\mathrm{MeC}_{6} \mathrm{H}_{4}$ & $\mathrm{Me}$ & $\mathrm{OMe}$ & 66 & 25 \\
3f & $3-\mathrm{BrC}_{6} \mathrm{H}_{4}$ & $\mathrm{Me}$ & $\mathrm{OMe}$ & 85 & 9 \\
3g & $2-$ thienyl $_{3}$ & $\mathrm{Me}$ & $\mathrm{OMe}$ & 59 & 34 \\
3h & $3,4-\left(\mathrm{OCH}_{2} \mathrm{O}_{6} \mathrm{C}_{6} \mathrm{H}_{3}\right.$ & $\mathrm{Me}$ & $\mathrm{OMe}$ & 65 & 26 \\
\hline
\end{tabular}

a All reactions were performed on a $1.0 \mathrm{mmol}$ scale.

b Yield of isolated product after column chromatography.
This can be explained by stereochemical outcome of the quinine square amide catalyst-based Michael additions of $\beta$-keto esters to nitroolefins, which is well known in this case. The relative and absolute configuration of trans-3g was determined as $2 S, 3 R, 4 R, 5 S$ by X-ray crystal-structure analysis (Figure 1$)^{10}$ showing a cis orientation between the ester and hydroxyl group and a trans relation between the ester and aryl substituent. No thermodynamic equilibrium between the hemiacetal and the open hydroxyl ketone was observed in NMR experiments. The relative configuration of all tetrahydropyranols $\mathbf{3 a}-\mathbf{h}$ was unambiguously determined by NOE measurements. They are all stable solids and can be easily recrystallized from benzene or methanol.

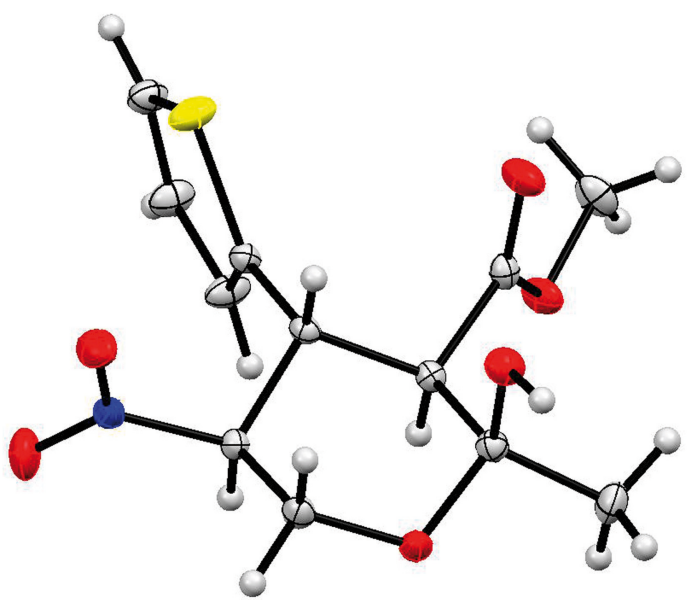

Figure 1 Absolute configuration of trans-3g confirmed by X-ray crystal-structure analysis ${ }^{10}$

Next various transformations of the enantiomerically pure domino product trans-3a were investigated to emphasize the value of this approach, for example, towards the synthesis of bioactive compounds such as pharmaceuticals (Scheme 2). It is well known that the hemiacetal unit can be reduced, methylated, or dehydrated to the corresponding tetrahydro- and dihydropyran derivatives. Therefore, 3a was synthesized, the epimers separated, and the trans3a was recrystallized from benzene in good yield (73\%). Then trans-3a was dehydrated to 4a in good yield (91\%) to obtain the virtually diastereo- and enantiomerically pure dihydropyran indicating that no epimerization or racemization had occurred. By treatment with $20 \mathrm{~mol} \%$ PTSA in $\mathrm{MeOH}$ for eight hours at room temperature trans-3a was converted to the acetal 7 in moderate yield (62\%), but again with excellent diastereo- $(98 \%)$ and enantiomeric excess (99\%). Compound trans-3a could be reduced to 8 with three equivalents $\mathrm{Et}_{3} \mathrm{SiH}$ in dichloromethane at $0{ }^{\circ} \mathrm{C}$ in moderate yield $(72 \%)$ and again with excellent diastereo- $(97 \%$ de) and enantiomeric excess ( $98 \%$ ee). The high stereoselectivity of our approaches is evident from the fact that only two diastereomers are formed while building up four contiguous stereocenters. The various transformations performed with the hemiace- 
tal underpin the stereochemical outcome of the first Michael reaction.

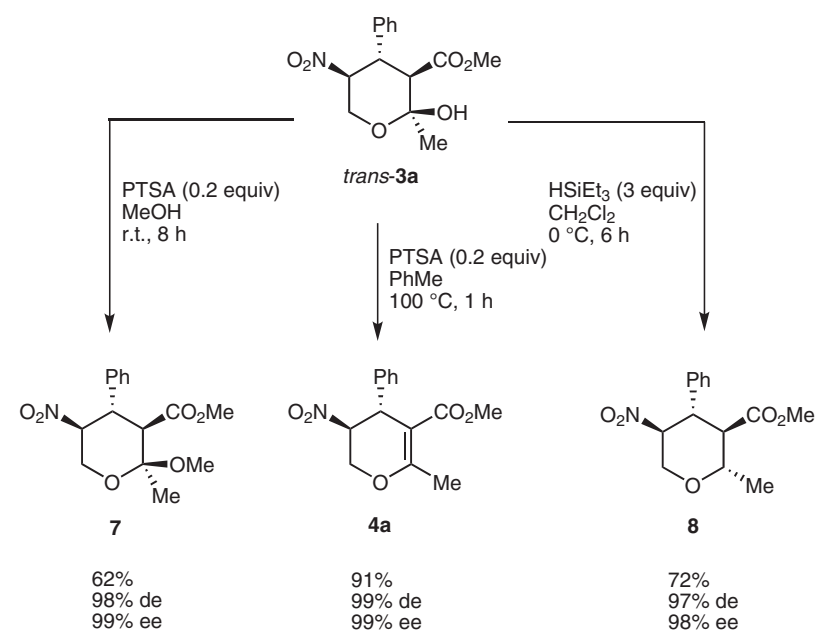

Scheme 2 Various synthetic transformations of enantiopure tetrahydropyranol trans-3a

In conclusion, we have developed an efficient diastereoand enantioselective asymmetric synthesis of polyfunctionalized dihydro- and tetrahydropyrans by employing a square amide-catalyzed domino Michael-hemiacetalization reaction. The new protocol allows several functional groups, such as the nitro, ester, ketone, and hydroxyl functions as well as substituents at the aromatic ring, attached to the hydropyran core. Subsequent transformations of the intermediate tetrahydropyranols gave acetals, dihydro$2 \mathrm{H}$-pyrans, and tetrahydro- $2 \mathrm{H}$-pyrans bearing two to four contiguous stereocenters. The title compounds are important heterocycles due to their widespread occurrence in nature and as privileged scaffolds in medicinal chemistry. They were obtained in very good yields and with good to excellent stereoselectivities.

Starting materials and reagents were purchased from commercial suppliers and used without further purification, unless stated otherwise. All solvents were dried by conventional methods. Preparative column chromatography: Merck silica gel 60, particle size 0.040 $0.063 \mathrm{~mm}$ (230-240 mesh, flash). Analytical TLC: silica gel 60 F254 plates from Merck, Darmstadt. Visualization of the developed TLC plates was performed by ultraviolet irradiation $(254 \mathrm{~nm})$ or by staining with a solution of $\mathrm{KMnO}_{4}$. Analytical HPLC was carried out on a Hewlett-Packard 1100 Series instrument using chiral stationary phases. ${ }^{1} \mathrm{H}$ and ${ }^{13} \mathrm{C}$ NMR spectra were recorded at r.t. on Varian Mercury 300, Varian Inova 400, or Varian Inova 600 instruments. Mass spectra were acquired on a Finnigan SSQ7000 (EI 70 eV) spectrometer, high-resolution mass spectra on a Finnigan MAT 95 and high-resolution ESI spectra on a Thermo Fisher Scientific LTQ-Orbitrap XL. IR spectra were recorded on a PerkinElmer 100 FT-IR Spectrum instrument. Microanalyses were performed with a Vario EL element analyzer. Melting points were determined with a Büchi melting point B-540 apparatus. Optical rotation values were measured on a Perkin-Elmer 241 polarimeter.

Domino Michael-Hemiacetalization Reaction; General Procedure 1 (GP1)

In a glass vial equipped with a magnetic stirring bar, catalyst $\mathbf{E}$ (10 mol\%) was added to a mixture of 1,3 dicarbonyl compound 1
$(1.0 \mathrm{mmol})$ and $(E)$-3-aryl-2-nitroprop-2-en-1-ol $2(1.0 \mathrm{mmol})$ in $\mathrm{CH}_{2} \mathrm{Cl}_{2}(4.0 \mathrm{~mL})$ at r.t. The reaction was monitored by TLC. After complete conversion of the starting material, the solvent was evaporated and the crude reaction mixture was purified by column chromatography affording products trans- and cis-3a-h, respectively, as pale yellow to colorless solids (Table 3).

One-Pot Domino Michael-Hemiacetalization and Dehydration Reaction; General Procedure 2 (GP2)

The tetrahydropyranols trans-/cis-3a-h were synthesized according GP1. After complete conversion of the starting material, the solvent was evaporated and toluene $(10 \mathrm{~mL})$ and PTSA $(20 \mathrm{~mol} \%)$ were added, and stirred for $1 \mathrm{~h}$ at $100{ }^{\circ} \mathrm{C}$. After complete conversion, the solvent was evaporated and the crude reaction mixture was purified by column chromatography affording products $\mathbf{4 a}-\mathbf{h}$ as pale yellow to colorless oils and as a cis/trans mixture (Table 2).

\section{Methyl (2S,3R,4S,5S)-2-Hydroxy-2-methyl-5-nitro-4-phen-}

yltetrahydro- $2 \mathrm{H}$-pyran-3-carboxylate (trans-3a)

Compound trans-3a was synthesized according to GP 1; yield: 238 $\mathrm{mg}(80 \%)$; colorless solid; $\mathrm{mp} 148{ }^{\circ} \mathrm{C}$ (benzene); $R_{f}=0.42$ ( $n$-pentane- $\left.\mathrm{Et}_{2} \mathrm{O}, 1: 1\right) ;[\alpha]_{\mathrm{D}}{ }^{20}-83.2$ (c 1.0, $\mathrm{CHCl}_{3}$ ); de $>95 \%$. The diastereomeric excess was determined by NMR spectroscopy.

IR (film): 3386 (vs), 1729 (vs), 1545 (vs), 1446 (s), 1355 (s), 1262 (m), $1180(\mathrm{~m}), 1115$ (vs), 1066 (vs), $887(\mathrm{~m}), 763(\mathrm{~s}), 695 \mathrm{~cm}^{-1}$ (s).

${ }^{1} \mathrm{H}$ NMR $\left(600 \mathrm{MHz}, \mathrm{CDCl}_{3}\right): \delta=1.48\left(\mathrm{~s}, 3 \mathrm{H}, \mathrm{CH}_{3}\right), 2.96(\mathrm{~d}$, $\left.J=12.4 \mathrm{~Hz}, 1 \mathrm{H}, \mathrm{CHCO}_{2} \mathrm{CH}_{3}\right), 3.44\left(\mathrm{~s}, 3 \mathrm{H}, \mathrm{CO}_{2} \mathrm{CH}_{3}\right), 3.87(\mathrm{~s}, 1 \mathrm{H}$, $\mathrm{OH}), 4.01(\mathrm{dd}, J=11.9,11.9 \mathrm{~Hz}, 1 \mathrm{H}, \mathrm{CHPh}), 4.11(\mathrm{dd}, J=10.9$, $\left.5.0 \mathrm{~Hz}, 1 \mathrm{H}, \mathrm{CH}_{2}\right), 4.49\left(\mathrm{dd}, J=10.9,10.9 \mathrm{~Hz}, 1 \mathrm{H}, \mathrm{CH}_{2}\right), 4.92$ (ddd, $\left.J=11.9,10.9,5.0 \mathrm{~Hz}, 1 \mathrm{H}, \mathrm{CHNO}_{2}\right), 7.22-7.32\left(\mathrm{~m}, 5 \mathrm{H}, \mathrm{C}_{6} \mathrm{H}_{5}\right)$.

${ }^{13} \mathrm{C}$ NMR $\left(151 \mathrm{MHz}, \mathrm{CDCl}_{3}\right): \delta=27.9\left(\mathrm{CH}_{3}\right), 43.8\left(\mathrm{CHCO}_{2} \mathrm{CH}_{3}\right)$, $52.3\left(\mathrm{CO}_{2} \mathrm{CH}_{3}\right), 55.2(\mathrm{CHPh}), 61.5\left(\mathrm{CH}_{2}\right), 85.6\left(\mathrm{CHNO}_{2}\right), 94.8$ $\left(\mathrm{C}_{\mathrm{q}} \mathrm{OH}\right), 127.8\left(2 \mathrm{CH}_{\mathrm{Ar}}\right), 128.4\left(\mathrm{CH}_{\mathrm{Ar}}\right), 129.0\left(2 \mathrm{CH}_{\mathrm{Ar}}\right), 135.7\left(\mathrm{C}_{\mathrm{Ar}}\right)$, $171.7\left(\mathrm{CO}_{2} \mathrm{CH}_{3}\right)$.

MS (EI, $70 \mathrm{eV}): m / z(\%)=295\left([\mathrm{M}]^{+}, 1\right), 278(28), 205(19), 189$ (27), 177 (54), 163 (70), 145 (56), 131 (100), 117 (51), 91 (27), 77 (23), 59 (23).

Anal. Calcd for $\mathrm{C}_{14} \mathrm{H}_{17} \mathrm{NO}_{6}$ : C, 56.94; $\mathrm{H}, 5.80 ; \mathrm{N}, 4.74$. Found: $\mathrm{C}$, 57.19; H, 5.84; N, 4.62.

Methyl (2S,3R,4S,5R)-2-Hydroxy-2-methyl-5-nitro-4-phenyltetrahydro-2H-pyran-3-carboxylate (cis-3a)

Compound cis-3a was synthesized according to GP 1; yield: $41 \mathrm{mg}$ $(14 \%)$; colorless solid; $\mathrm{mp} 56{ }^{\circ} \mathrm{C}(\mathrm{MeOH}) ; R_{f}=0.12$ (n-pentane$\left.\mathrm{Et}_{2} \mathrm{O}, 1: 1\right) ;[\alpha]_{\mathrm{D}}{ }^{20}-11.0\left(c 0.5, \mathrm{CHCl}_{3}\right) ; \mathrm{de}>95 \%$. The diastereomeric excess was determined by NMR spectroscopy.

${ }^{1} \mathrm{H}$ NMR $\left(600 \mathrm{MHz}, \mathrm{CDCl}_{3}\right): \delta=2.24\left(\mathrm{~s}, 3 \mathrm{H}, \mathrm{CH}_{3}\right), 3.35(\mathrm{~s}, 1 \mathrm{H}$, $\mathrm{OH}), 3.55\left(\mathrm{~s}, 3 \mathrm{H}, \mathrm{CO}_{2} \mathrm{CH}_{3}\right), 3.87(\mathrm{dd}, J=12.4,4.0 \mathrm{~Hz}, 1 \mathrm{H}, \mathrm{CHPh})$, $4.10\left(\mathrm{~d}, J=12.4 \mathrm{~Hz}, 1 \mathrm{H}, \mathrm{CHCO}_{2} \mathrm{CH}_{3}\right), 4.14(\mathrm{dd}, J=13.4,3.5 \mathrm{~Hz}$, $\left.1 \mathrm{H}, \mathrm{CH}_{2}\right), 4.58\left(\mathrm{dd}, J=13.4,3.0 \mathrm{~Hz}, 1 \mathrm{H}, \mathrm{CH}_{2}\right), 4.79$ (ddd, $J=4.0$, 3.5, 3.0 Hz, $\left.1 \mathrm{H}, \mathrm{CHNO}_{2}\right), 7.22-7.34\left(\mathrm{~m}, 5 \mathrm{H}, \mathrm{C}_{6} \mathrm{H}_{5}\right)$.

${ }^{13} \mathrm{C} \mathrm{NMR}\left(151 \mathrm{MHz}, \mathrm{CDCl}_{3}\right): \delta=28.6\left(\mathrm{CH}_{3}\right), 41.1\left(\mathrm{CHCO}_{2} \mathrm{CH}_{3}\right)$, $48.0\left(\mathrm{CO}_{2} \mathrm{CH}_{3}\right), 52.4(\mathrm{CHPh}), 61.8\left(\mathrm{CH}_{2}\right), 84.7\left(\mathrm{CHNO}_{2}\right), 94.2$ $\left(\mathrm{C}_{\mathrm{q}} \mathrm{OH}\right), 127.8\left(2 \mathrm{CH}_{\mathrm{Ar}}\right), 128.2\left(\mathrm{CH}_{\mathrm{Ar}}\right), 128.9\left(2 \mathrm{CH}_{\mathrm{Ar}}\right), 136.1\left(\mathrm{C}_{\mathrm{Ar}}\right)$, $172.9\left(\mathrm{CO}_{2} \mathrm{CH}_{3}\right)$.

Ethyl (2S,3R,4S,5S)-2-Hydroxy-2-methyl-5-nitro-4-phenyltetrahydro-2H-pyran-3-carboxylate (trans-3b)

Compound trans-3b was synthesized according to GP 1; yield: 238 $\mathrm{mg}\left(77 \%\right.$ ); colorless solid (benzene); $\mathrm{mp} 112^{\circ} \mathrm{C} ; R_{f}=0.39$ ( $n$-pentane- $\left.\mathrm{Et}_{2} \mathrm{O}, 1: 1\right) ;[\alpha]_{\mathrm{D}}{ }^{20}-65.8\left(c 1.0, \mathrm{CHCl}_{3}\right)$; de $>95 \%$. The diastereomeric excess was determined by NMR spectroscopy.

IR (film): 3449 (s), 1718 (vs), 1547 (vs), 1456 (m), 1381 (vs), 1261 (m), 1179 (m), 1111 (vs), 1069 (vs), 881 (vs), 762 (s), $700 \mathrm{~cm}^{-1}$ (s).

${ }^{1} \mathrm{H} \mathrm{NMR}\left(600 \mathrm{MHz}, \mathrm{CDCl}_{3}\right): \delta=0.83\left(\mathrm{t}, J=7.4 \mathrm{~Hz}, 3 \mathrm{H}, \mathrm{CH}_{2} \mathrm{CH}_{3}\right)$, $1.47\left(\mathrm{~s}, 3 \mathrm{H}, \mathrm{CH}_{3}\right), 2.92\left(\mathrm{~d}, J=12.4 \mathrm{~Hz}, 1 \mathrm{H}, \mathrm{CHCO}_{2} \mathrm{Et}\right), 3.89(\mathrm{dq}$, $\left.J=7.4,2.5 \mathrm{~Hz}, 2 \mathrm{H}, \mathrm{CH}_{2} \mathrm{CH}_{3}\right), 3.98(\mathrm{dd}, J=11.9,11.9 \mathrm{~Hz}, 1 \mathrm{H}$, 
$\mathrm{CHPh}), 4.09$ (s, $1 \mathrm{H}, \mathrm{OH}), 4.11\left(\mathrm{dd}, J=10.9,5.0 \mathrm{~Hz}, 1 \mathrm{H}, \mathrm{CH}_{2}\right)$, 4.49 (dd, $\left.J=10.9,10.9 \mathrm{~Hz}, 1 \mathrm{H}, \mathrm{CH}_{2}\right), 4.92$ (ddd, $J=11.9,10.9,5.0$ $\left.\mathrm{Hz}, 1 \mathrm{H}, \mathrm{CHNO}_{2}\right), 7.20-7.32\left(\mathrm{~m}, 5 \mathrm{H}, \mathrm{C}_{6} \mathrm{H}_{5}\right)$.

${ }^{13} \mathrm{C} \mathrm{NMR}\left(151 \mathrm{MHz}, \mathrm{CDCl}_{3}\right): \delta=13.6\left(\mathrm{CH}_{2} \mathrm{CH}_{3}\right), 27.8\left(\mathrm{CH}_{3}\right), 44.1$ $(\mathrm{CHCO}=\mathrm{Et}), 55.0(\mathrm{CHPh}), 61.5\left(\mathrm{CH}_{2}\right), 61.6\left(\mathrm{CH}_{2}\right), 85.6\left(\mathrm{CHNO}_{2}\right)$, $94.7\left(\mathrm{C}_{\mathrm{q}} \mathrm{OH}\right), 128.0\left(2 \mathrm{CH}_{\mathrm{Ar}}\right), 128.4\left(\mathrm{CH}_{\mathrm{Ar}}\right), 128.9\left(2 \mathrm{CH}_{\mathrm{Ar}}\right), 135.6$ $\left(\mathrm{C}_{\mathrm{Ar}}\right), 171.8\left(\mathrm{CO}_{2} \mathrm{Et}\right)$.

MS (EI, $70 \mathrm{eV}): m / z(\%)=292\left(\left[\mathrm{M}-\mathrm{H}_{2} \mathrm{O}\right]^{+}, 6\right), 219(10), 203(10)$ 191 (31), 177 (89), 173 (23), 157 (21), 149 (16), 145 (51), 131 (100), 117 (52), 103 (37), 91 (39), 77 (24), 51 (23).

HRMS: $m / z$ calcd for $\mathrm{C}_{15} \mathrm{H}_{19} \mathrm{NO}_{6}+\mathrm{Na}$ : 332.1110; found: 332.1095 .

\section{Ethyl $(2 S, 3 R, 4 S, 5 R)$-2-Hydroxy-2-methyl-5-nitro-4-phenyltet-} rahydro-2 $H$-pyran-3-carboxylate (cis-3b)

Compound cis-3b was synthesized according to GP 1; yield: $56 \mathrm{mg}$ (18\%); colorless solid; $\mathrm{mp} 42{ }^{\circ} \mathrm{C}$ (benzene); $R_{f}=0.21$ ( $n$-pentane$\left.\mathrm{Et}_{2} \mathrm{O}, 1: 1\right) ;[\alpha]_{\mathrm{D}}{ }^{20}-9.1\left(c 0.5, \mathrm{CHCl}_{3}\right) ; \mathrm{de}>95 \%$. The diastereomeric excess was determined by NMR spectroscopy.

${ }^{1} \mathrm{H} \mathrm{NMR}\left(600 \mathrm{MHz}, \mathrm{CDCl}_{3}\right): \delta=0.97\left(\mathrm{t}, J=6.9 \mathrm{~Hz}, 3 \mathrm{H}, \mathrm{CH}_{2} \mathrm{CH}_{3}\right)$, $1.62\left(\mathrm{~s}, 3 \mathrm{H}, \mathrm{CH}_{3}\right), 3.59(\mathrm{~s}, 1 \mathrm{H}, \mathrm{OH}), 3.84(\mathrm{dd}, J=12.9,4.5 \mathrm{~Hz}, 1$ $\mathrm{H}, \mathrm{CHPh}), 4.01\left(\mathrm{q}, J=6.9 \mathrm{~Hz}, 2 \mathrm{H}, \mathrm{CH}_{2} \mathrm{CH}_{3}\right), 4.06(\mathrm{~d}, J=12.9 \mathrm{~Hz}$ $\left.1 \mathrm{H}, \mathrm{CHCO}_{2} \mathrm{Et}\right), 4.13\left(\mathrm{dd}, J=13.4,1.0 \mathrm{~Hz}, 1 \mathrm{H}, \mathrm{CH}_{2}\right), 4.59(\mathrm{dd}$, $\left.J=13.4,3.0 \mathrm{~Hz}, 1 \mathrm{H}, \mathrm{CH}_{2}\right), 4.78(\mathrm{ddd}, J=4.5,3.0,1.0 \mathrm{~Hz}, 1 \mathrm{H}, \mathrm{CH}-$ $\left.\mathrm{NO}_{2}\right), 7.20-7.32\left(\mathrm{~m}, 5 \mathrm{H}, \mathrm{C}_{6} \mathrm{H}_{5}\right)$.

${ }^{13} \mathrm{C}$ NMR (151 MHz, $\left.\mathrm{CDCl}_{3}\right): \delta=13.7\left(\mathrm{CH}_{2} \mathrm{CH}_{3}\right), 28.5\left(\mathrm{CH}_{3}\right), 41.3$ $(\mathrm{CHPh}), 48.0\left(\mathrm{CHCO}_{2} \mathrm{Et}\right), 61.4\left(\mathrm{CH}_{2} \mathrm{CH}_{3}\right), 61.8\left(\mathrm{CH}_{2}\right), 84.6(\mathrm{CH}-$ $\left.\mathrm{NO}_{2}\right), 95.1\left(\mathrm{C}_{\mathrm{q}} \mathrm{OH}\right), 128.1\left(\mathrm{CH}_{\mathrm{Ar}}\right), 128.2\left(\mathrm{CH}_{\mathrm{Ar}}\right), 128.3\left(\mathrm{CH}_{\mathrm{Ar}}\right)$, $128.8\left(2 \mathrm{CH}_{\mathrm{Ar}}\right), 136.0\left(\mathrm{C}_{\mathrm{Ar}}\right), 172.7\left(\mathrm{CO}_{2} \mathrm{Et}\right)$.

\section{Ethyl $(2 S, 3 R, 4 S, 5 S)$-2-Hydroxy-5-nitro-2,4-diphenyltetrahy-} dro-2H-pyran-3-carboxylate (trans-3c)

Compound trans-3c was synthesized according to GP 1 to yield 338 mg $(91 \%)$ of a colorless solid; $\mathrm{mp} 156{ }^{\circ} \mathrm{C}$ (benzene); $R_{f}=0.44(n$ pentane- $\left.\mathrm{Et}_{2} \mathrm{O}, 1: 1\right) ;[\alpha]_{\mathrm{D}}{ }^{20}-113.3\left(c 1.0, \mathrm{CHCl}_{3}\right)$; de $>95 \%$. The diastereomeric excess was determined by NMR spectroscopy.

IR (film): 3432 (s), 1727 (vs), 1546 (vs), 1453 (m), 1356 (vs), 1275 (m), 1177 (s), 1110 (s), 1055 (vs), 981 (vs), 760 (s), $701 \mathrm{~cm}^{-1}$ (s).

${ }^{1} \mathrm{H} \mathrm{NMR}\left(600 \mathrm{MHz}, \mathrm{CDCl}_{3}\right): \delta=0.57\left(\mathrm{t}, J=6.9 \mathrm{~Hz}, 3 \mathrm{H}, \mathrm{CH}_{2} \mathrm{CH}_{3}\right)$, $3.16\left(\mathrm{~d}, J=12.4 \mathrm{~Hz}, 1 \mathrm{H}, \mathrm{CHCO}_{2} \mathrm{Et}\right), 3.62(\mathrm{q}, J=6.9 \mathrm{~Hz}, 2 \mathrm{H}$ $\left.\mathrm{CH}_{2} \mathrm{CH}_{3}\right), 4.15$ (dd, $\left.J=12.4,11.4 \mathrm{~Hz}, 1 \mathrm{H}, \mathrm{CHPh}\right), 4.32(\mathrm{dd}$ $\left.J=10.9,5.0 \mathrm{~Hz}, 1 \mathrm{H}, \mathrm{CH}_{2}\right), 4.73\left(\mathrm{dd}, J=10.9,10.9 \mathrm{~Hz}, 1 \mathrm{H}, \mathrm{CH}_{2}\right)$, $5.00(\mathrm{~s}, 1 \mathrm{H}, \mathrm{OH}), 5.12\left(\mathrm{ddd}, J=11.4,10.9,5.0 \mathrm{~Hz}, 1 \mathrm{H}, \mathrm{CHNO}_{2}\right)$, 7.23-7.55 (m, $\left.10 \mathrm{H}, \mathrm{C}_{6} \mathrm{H}_{5}\right)$.

${ }^{13} \mathrm{C} \mathrm{NMR}\left(151 \mathrm{MHz}, \mathrm{CDCl}_{3}\right): \delta=13.3\left(\mathrm{CH}_{2} \mathrm{CH}_{3}\right), 44.8\left(\mathrm{CHCO}_{2} \mathrm{Et}\right)$ 56.6 $(\mathrm{CHPh}), 61.4\left(\mathrm{CH}_{2}\right), 62.1\left(\mathrm{CH}_{2}\right), 85.5\left(\mathrm{CHNO}_{2}\right), 96.0\left(\mathrm{C}_{\mathrm{q}} \mathrm{OH}\right)$, $125.4\left(2 \mathrm{CH}_{\mathrm{Ar}}\right), 127.9\left(\mathrm{CH}_{\mathrm{Ar}}\right), 128.4\left(\mathrm{CH}_{\mathrm{Ar}}\right), 128.6\left(2 \mathrm{CH}_{\mathrm{Ar}}\right), 128.7$ $\left(2 \mathrm{CH}_{\mathrm{Ar}}\right), 128.9\left(2 \mathrm{CH}_{\mathrm{Ar}}\right), 135.2\left(\mathrm{C}_{\mathrm{Ar}}\right), 141.1\left(\mathrm{C}_{\mathrm{Ar}}\right), 171.6\left(\mathrm{CO}_{2} \mathrm{Et}\right)$.

MS (EI, $70 \mathrm{eV}): m / z(\%)=354\left(\left[\mathrm{M}-\mathrm{H}_{2} \mathrm{O}\right]^{+}, 2\right), 131(8), 105(100)$ 77 (26).

Anal. Calcd for $\mathrm{C}_{20} \mathrm{H}_{21} \mathrm{NO}_{6}$ : C, 64.68; $\mathrm{H}, 5.70 ; \mathrm{N}, 3.77$. Found: $\mathrm{C}$, 64.77; H, 5.62; N, 3.66 .

1-[(2S,3S,4S,5S)-2-Hydroxy-2-methyl-5-nitro-4-phenyltetrahydro-2H-pyran-3-yl]ethanone (trans-3d)

Compound trans-3d was synthesized according to GP 1; yield; 223 mg (80\%); colorless solid; mp $98{ }^{\circ} \mathrm{C}$ (benzene); $R_{f}=0.21$ ( $n$-pentane- $\left.\mathrm{Et}_{2} \mathrm{O}, 1: 1\right) ;[\alpha]_{\mathrm{D}}{ }^{20}-54.4\left(c 1.0, \mathrm{CHCl}_{3}\right)$; de $>95 \%$. The diastereomeric excess was determined by NMR spectroscopy.

IR (film): 3502 (s), 3413 (s), 1695 (vs), 15498 (vs), 1361 (vs), 1233 (w), 1123 (vs), 1051 (vs), $929(\mathrm{~m}), 885$ (m), 752 (s), $702 \mathrm{~cm}^{-1}$ (s).

${ }^{1} \mathrm{H}$ NMR (600 MHz, $\left.\mathrm{CDCl}_{3}\right): \delta=1.41\left(\mathrm{~s}, 3 \mathrm{H}, \mathrm{CH}_{3}\right), 1.77(\mathrm{~s}, 3 \mathrm{H}$, $\mathrm{CH}_{3}$ ), 3.12 (d, $\left.J=12.4 \mathrm{~Hz}, 1 \mathrm{H}, \mathrm{CHCOCH}_{3}\right), 3.92$ (dd, $J=11.9$, $11.9 \mathrm{~Hz}, 1 \mathrm{H}, \mathrm{CHPh}), 4.11\left(\mathrm{dd}, J=10.4,5.0 \mathrm{~Hz}, 1 \mathrm{H}, \mathrm{CH}_{2}\right), 4.32(\mathrm{~s}$, $1 \mathrm{H}, \mathrm{OH}), 4.49$ (dd, $\left.J=10.9,10.4 \mathrm{~Hz}, 1 \mathrm{H}, \mathrm{CH}_{2}\right), 4.95$ (ddd, $\left.J=11.9,10.9,5.0 \mathrm{~Hz}, 1 \mathrm{H}, \mathrm{CHNO}_{2}\right), 7.19-7.36\left(\mathrm{~m}, 5 \mathrm{H}, \mathrm{C}_{6} \mathrm{H}_{5}\right)$. $\left.{ }^{13} \mathrm{C} \mathrm{NMR}\left(151 \mathrm{MHz}, \mathrm{CDCl}_{3}\right): \delta=27.9 \mathrm{CH}_{3}\right), 32.7\left(\mathrm{COCH}_{3}\right), 44.5$ $(\mathrm{CHPh}), 60.3(\mathrm{CHCOMe}), 61.5\left(\mathrm{CH}_{2}\right), 85.6\left(\mathrm{CHNO}_{2}\right), 94.8$ $\left(\mathrm{C}_{\mathrm{q}} \mathrm{OH}\right), 127.8\left(2 \mathrm{CH}_{\mathrm{Ar}}\right), 128.7\left(\mathrm{CH}_{\mathrm{Ar}}\right), 129.4\left(2 \mathrm{CH}_{\mathrm{Ar}}\right), 135.5\left(\mathrm{C}_{\mathrm{Ar}}\right)$, $210.9(\mathrm{COMe})$.

MS (CI, $70 \mathrm{eV}): m / z(\%)=262\left(\left[\mathrm{M}-\mathrm{H}_{2} \mathrm{O}\right]^{+}, 100\right), 220(50), 189$ (15), 173 (64), 147 (48), 131 (21), 91 (13).

Anal. Calcd for $\mathrm{C}_{14} \mathrm{H}_{17} \mathrm{NO}_{5}: \mathrm{C}, 60.21 ; \mathrm{H}, 6.14 ; \mathrm{N}, 5.02$. Found: $\mathrm{C}$, $60.27 ; \mathrm{H}, 6.09 ; \mathrm{N}, 4.90$

1-[(2S,3S,4S,5R)-2-Hydroxy-2-methyl-5-nitro-4-phenyltetrahydro-2H-pyran-3-yl]ethanone (cis-3d)

Compound cis-3d was synthesized according to GP 1; yield: $17 \mathrm{mg}$ $(6 \%)$; colorless solid $(\mathrm{MeOH}) ; \mathrm{mp} 40{ }^{\circ} \mathrm{C} ; R_{f}=0.23$ (n-pentane$\left.\mathrm{Et}_{2} \mathrm{O}, 1: 2\right) ;[\alpha]_{\mathrm{D}}{ }^{20}-6.2\left(c 0.2, \mathrm{CHCl}_{3}\right) ;$ de $>95 \%$. The diastereomeric excess was determined by NMR spectroscopy.

${ }^{1} \mathrm{H}$ NMR $\left(600 \mathrm{MHz}, \mathrm{CDCl}_{3}\right): \delta=1.56\left(\mathrm{~s}, 3 \mathrm{H}, \mathrm{CH}_{3}\right), 2.08(\mathrm{~s}, 3 \mathrm{H}$, $\left.\mathrm{CH}_{3}\right), 3.68(\mathrm{~s}, 1 \mathrm{H}, \mathrm{OH}), 3.79(\mathrm{dd}, J=12.9,4.0 \mathrm{~Hz}, 1 \mathrm{H}, \mathrm{CHPh})$, $4.13\left(\mathrm{~d}, J=13.4 \mathrm{~Hz}, 1 \mathrm{H}, \mathrm{CH}_{2}\right), 4.27(\mathrm{~d}, J=12.4 \mathrm{~Hz}, 1 \mathrm{H}, \mathrm{CH}-$ $\mathrm{COMe}), 4.58\left(\mathrm{dd}, J=13.4,3.0 \mathrm{~Hz}, 1 \mathrm{H}, \mathrm{CH}_{2}\right), 4.76(\mathrm{~m}, 1 \mathrm{H}, \mathrm{CH}-$ $\left.\mathrm{NO}_{2}\right), 7.20-7.24\left(\mathrm{~m}, 2 \mathrm{H}, \mathrm{CH}_{\mathrm{Ar}}\right), 7.29-7.34\left(\mathrm{~m}, 3 \mathrm{H}, \mathrm{CH}_{\mathrm{Ar}}\right)$.

${ }^{13} \mathrm{C} \mathrm{NMR}\left(151 \mathrm{MHz}, \mathrm{CDCl}_{3}\right): \delta=28.7\left(\mathrm{CH}_{3}\right), 32.5\left(\mathrm{COCH}_{3}\right), 42.0$ $(\mathrm{CHPh}), 54.0(\mathrm{CHCOMe}), 61.7\left(\mathrm{CH}_{2}\right), 84.8\left(\mathrm{CHNO}_{2}\right), 95.0$ $\left(\mathrm{C}_{\mathrm{q}} \mathrm{OH}\right), 128.1\left(2 \mathrm{CH}_{\mathrm{Ar}}\right), 128.6\left(\mathrm{CH}_{\mathrm{Ar}}\right), 129.2\left(2 \mathrm{CH}_{\mathrm{Ar}}\right), 135.7\left(\mathrm{C}_{\mathrm{Ar}}\right)$, $211.5(\mathrm{COMe})$.

\section{Methyl $(2 S, 3 R, 4 S, 5 S)-2-H y d r o x y-2-m e t h y l-5-n i t r o-4-(p-$} tolyl)tetrahydro-2H-pyran-3-carboxylate (trans-3e)

Compound trans-3e was synthesized according to GP 1; yield: 204 $\mathrm{mg}(66 \%)$; colorless solid; (benzene); $\mathrm{mp} 151{ }^{\circ} \mathrm{C} ; R_{f}=0.38$ ( $n$-pentane- $\left.\mathrm{Et}_{2} \mathrm{O}, 1: 1\right) ;[\alpha]_{\mathrm{D}}{ }^{20}-85.3$ (c 1.0, $\mathrm{CHCl}_{3}$ ); de $>95 \%$. The diastereomeric excess was determined by NMR spectroscopy.

IR (film): 3508 (vs), 2998 (w), 1716 (vs), 1543 (vs), 1442 (m), 1365 (vs), 1217 (m), 1120 (vs), 1066 (vs), $879(\mathrm{~m}), 809(\mathrm{~m}), 727 \mathrm{~cm}^{-1}$ (s).

${ }^{1} \mathrm{H}$ NMR $\left(600 \mathrm{MHz}, \mathrm{CDCl}_{3}\right): \delta=1.48\left(\mathrm{~s}, 3 \mathrm{H}, \mathrm{CH}_{3}\right), 2.29(\mathrm{~s}, 3 \mathrm{H}, p-$ $\left.\mathrm{CH}_{3}\right), 2.94\left(\mathrm{~d}, J=12.4 \mathrm{~Hz}, 1 \mathrm{H}, \mathrm{CHCO}_{2} \mathrm{CH}_{3}\right), 3.44(\mathrm{~s}, 3 \mathrm{H}$, $\left.\mathrm{CO}_{2} \mathrm{CH}_{3}\right), 3.85(\mathrm{~s}, 1 \mathrm{H}, \mathrm{OH}), 3.97(\mathrm{dd}, J=11.9,11.9 \mathrm{~Hz}, 1 \mathrm{H}$, CHAr), 4.09 (dd, $\left.J=10.4,5.0 \mathrm{~Hz}, 1 \mathrm{H}, \mathrm{CH}_{2}\right), 4.47$ (dd, $J=10.9$, $\left.10.4 \mathrm{~Hz}, 1 \mathrm{H}, \mathrm{CH}_{2}\right), 4.88$ (ddd, $J=11.9,10.9,5.0 \mathrm{~Hz}, 1 \mathrm{H}, \mathrm{CHNO}_{2}$ ), 7.07-7.13 (m, $\left.4 \mathrm{H}, \mathrm{CH}_{\mathrm{Ar}}\right)$.

${ }^{13} \mathrm{C} \mathrm{NMR}\left(151 \mathrm{MHz}, \mathrm{CDCl}_{3}\right): \delta=21.1\left(p-\mathrm{CH}_{3}\right), 28.0\left(\mathrm{CH}_{3}\right), 43.5$ $\left(\mathrm{CHCO}_{2} \mathrm{CH}_{3}\right), 52.3\left(\mathrm{CO}_{2} \mathrm{CH}_{3}\right), 55.2(\mathrm{CHAr}), 61.6\left(\mathrm{CH}_{2}\right), 85.7(\mathrm{CH}-$ $\left.\mathrm{NO}_{2}\right), 94.8\left(\mathrm{C}_{\mathrm{q}} \mathrm{OH}\right), 127.6\left(2 \mathrm{CH}_{\mathrm{Ar}}\right), 129.7\left(2 \mathrm{CH}_{\mathrm{Ar}}\right), 132.6\left(\mathrm{C}_{\mathrm{Ar}}\right)$, $138.1\left(\mathrm{C}_{\mathrm{Ar}}\right), 172.0\left(\mathrm{CO}_{2} \mathrm{CH}_{3}\right)$.

MS (EI, $70 \mathrm{eV}): m / z(\%)=309\left(\left[\mathrm{M}^{+}\right], 14\right), 292(100), 219(18), 203$ (20), 191 (18), 185 (22), 176 (26), 159 (10), 145 (19).

HRMS: $m / z$ calcd for $\mathrm{C}_{15} \mathrm{H}_{19} \mathrm{NO}_{6}+\mathrm{Na}: 332.1105$; found: 332.1105 . Anal. Calcd for $\mathrm{C}_{15} \mathrm{H}_{19} \mathrm{NO}_{6}$ : C, 58.25; H, 6.19; N, 4.53. Found: $\mathrm{C}$, 58.17; H, 6.28; N, 4.82 .

\section{Methyl (2S,3R,4S,5R)-2-Hydroxy-2-methyl-5-nitro-4-( $p$ - tolyl)tetrahydro-2H-pyran-3-carboxylate (cis-3e)} Compound cis-3e was synthesized according to GP 1 ; yield: $77 \mathrm{mg}$ (25\%); colorless solid (benzene); mp $71 \mathrm{C} ; R_{f}=0.31$ (n-pentane$\left.\mathrm{Et}_{2} \mathrm{O}, 1: 2\right) ;[\alpha]_{\mathrm{D}}{ }^{20}-7.1\left(c 0.5, \mathrm{CHCl}_{3}\right) ; \mathrm{de}>95 \%$. The diastereomeric excess was determined by NMR spectroscopy.

${ }^{1} \mathrm{H}$ NMR $\left(600 \mathrm{MHz}, \mathrm{CDCl}_{3}\right): \delta=1.61\left(\mathrm{~s}, 3 \mathrm{H}, \mathrm{CH}_{3}\right), 2.29(\mathrm{~s}, 3 \mathrm{H}, p-$ $\left.\mathrm{CH}_{3}\right), 3.36(\mathrm{~s}, 1 \mathrm{H}, \mathrm{OH}), 3.56\left(\mathrm{~s}, 3 \mathrm{H}, \mathrm{CO}_{2} \mathrm{CH}_{3}\right), 3.83(\mathrm{dd}, J=12.9$, $4.5 \mathrm{~Hz}, 1 \mathrm{H}, \mathrm{CHAr}), 4.07$ (d, $\left.J=12.9 \mathrm{~Hz}, 1 \mathrm{H}, \mathrm{CHCO}_{2} \mathrm{CH}_{3}\right), 4.13$ $\left(\mathrm{dd}, J=13.4,1.0 \mathrm{~Hz}, 1 \mathrm{H}, \mathrm{CH}_{2}\right), 4.57(\mathrm{dd}, J=13.4,3.0 \mathrm{~Hz}, 1 \mathrm{H}$, $\left.\mathrm{CH}_{2}\right), 4.76$ (ddd, $\left.J=4.5,3.0,1.0 \mathrm{~Hz}, 1 \mathrm{H}, \mathrm{CHNO}_{2}\right), 7.08-7.12(\mathrm{~m}$, $\left.4 \mathrm{H}, \mathrm{CH}_{\mathrm{Ar}}\right)$.

${ }^{13} \mathrm{C} \mathrm{NMR}\left(151 \mathrm{MHz}, \mathrm{CDCl}_{3}\right): \delta=21.0\left(p-\mathrm{CH}_{3}\right), 28.6\left(\mathrm{CH}_{3}\right), 40.7$ $\left(\mathrm{CHCO}_{2} \mathrm{CH}_{3}\right), 48.0(\mathrm{CHAr}), 52.3\left(\mathrm{CO}_{2} \mathrm{CH}_{3}\right), 61.7\left(\mathrm{CH}_{2}\right), 84.8(\mathrm{CH}-$ $\left.\mathrm{NO}_{2}\right)$, $95.1\left(\mathrm{C}_{\mathrm{q}} \mathrm{OH}\right), 127.6\left(2 \mathrm{CH}_{\mathrm{Ar}}\right), 129.6\left(2 \mathrm{CH}_{\mathrm{Ar}}\right), 133.0\left(\mathrm{C}_{\mathrm{Ar}}\right)$, $137.8\left(\mathrm{C}_{\mathrm{Ar}}\right), 173.0\left(\mathrm{CO}_{2} \mathrm{CH}_{3}\right)$. 
Methyl (2S,3R,4S,5S)-4-(3-Bromophenyl)-2-hydroxy-2-methyl5-nitrotetrahydro-2H-pyran-3-carboxylate (trans-3f) Compound trans-3f was synthesized according to GP 1; yield: 318 $\mathrm{mg}(85 \%)$; colorless solid; $\mathrm{mp} 158{ }^{\circ} \mathrm{C}$ (benzene); $R_{f}=0.33$ ( $n$-pentane-Et $\left.{ }_{2} \mathrm{O}, 1: 1\right) ;[\alpha]_{\mathrm{D}}{ }^{20}-48.4\left(c 1.0, \mathrm{CHCl}_{3}\right) ; \mathrm{de}>95 \%$. The diastereomeric excess was determined by NMR spectroscopy.

IR (film): 3556 (s), 1725 (vs), 1547 (vs), 1436 (m), 1363 (vs), 1263 (m), 1120 (vs), 1066 (vs), 885 (m), 842 (m), 787 (s), 691 (s), 576 (s), $536 \mathrm{~cm}^{-1}$ (s).

${ }^{1} \mathrm{H}$ NMR $\left(600 \mathrm{MHz}, \mathrm{CDCl}_{3}\right): \delta=1.48\left(\mathrm{~s}, 3 \mathrm{H}, \mathrm{CH}_{3}\right), 2.92(\mathrm{~d}$, $\left.J=12.4 \mathrm{~Hz}, 1 \mathrm{H}, \mathrm{CHCO} \mathrm{CH}_{3}\right), 3.48\left(\mathrm{~s}, 3 \mathrm{H}, \mathrm{CO}_{2} \mathrm{CH}_{3}\right), 3.72(\mathrm{~s}, 1 \mathrm{H}$ $\mathrm{OH}), 4.09$ (dd, $J=12.4,11.9 \mathrm{~Hz}, 1 \mathrm{H}, \mathrm{CHAr}), 4.11$ (dd, $J=10.4$, $\left.5.0 \mathrm{~Hz}, 1 \mathrm{H}, \mathrm{CH}_{2}\right), 4.45\left(\mathrm{dd}, J=10.9,10.4 \mathrm{~Hz}, 1 \mathrm{H}, \mathrm{CH}_{2}\right), 4.88(\mathrm{ddd}$, $\left.J=11.9,10.9,5.0 \mathrm{~Hz}, 1 \mathrm{H}, \mathrm{CHNO}_{2}\right), 7.16-7.20\left(\mathrm{~m}, 2 \mathrm{H}, \mathrm{CH}_{\mathrm{Ar}}\right)$, 7.35-7.42 (m, $\left.1 \mathrm{H}, \mathrm{CH}_{\mathrm{Ar}}\right)$.

${ }^{13} \mathrm{C}$ NMR (151 MHz, $\left.\mathrm{CDCl}_{3}\right): \delta=27.9\left(\mathrm{CH}_{3}\right), 43.2(\mathrm{CHAr}), 52.5$ $\left(\mathrm{CO}_{2} \mathrm{CH}_{3}\right), 55.0\left(\mathrm{CHCO}_{2} \mathrm{CH}_{3}\right), 61.4\left(\mathrm{CH}_{2}\right), 85.2\left(\mathrm{CHNO}_{2}\right), 94.8$ $\left(\mathrm{C}_{\mathrm{q}} \mathrm{OH}\right), 123.0\left(\mathrm{C}_{\mathrm{Ar}}\right), 126.7\left(\mathrm{CH}_{\mathrm{Ar}}\right), 130.5\left(\mathrm{CH}_{\mathrm{Ar}}\right), 130.8\left(\mathrm{CH}_{\mathrm{Ar}}\right)$, $131.6\left(\mathrm{CH}_{\mathrm{Ar}}\right), 138.8\left(\mathrm{C}_{\mathrm{Ar}}\right), 171.3\left(\mathrm{CO}_{2} \mathrm{CH}_{3}\right)$.

MS (EI, $70 \mathrm{eV}): m / z(\%)=356\left([\mathrm{M}]^{+}, 7\right), 267(20), 257(26), 255$ (30), 241 (35) 225 (43), 213 (39), 211 (62), 195 (25), 183 (15), 155 (34), 128 (100), $116(55), 102$ (48), 77 (15), 59 (28).

HRMS: $m / z$ calcd for $\mathrm{C}_{14} \mathrm{H}_{16} \mathrm{BrNO}_{6}+\mathrm{Na}$ : 398.0038; found: 398.0718 .

Methyl (2S,3R,4S,5R)-4-(3-Bromophenyl)-2-hydroxy-2-methyl5-nitrotetrahydro-2H-pyran-3-carboxylate (cis-3f)

Compound cis-3f was synthesized according to GP 1; yield: $34 \mathrm{mg}$ (9\%); colorless solid (benzene); mp $88^{\circ} \mathrm{C} ; R_{f}=0.29$ (n-pentane$\left.\mathrm{Et}_{2} \mathrm{O}, 1: 2\right) ;[\alpha]_{\mathrm{D}}^{20}-5.1\left(\mathrm{c} 0.2, \mathrm{CHCl}_{3}\right)$; de $>95 \%$. The diastereomeric excess was determined by NMR spectroscopy.

${ }^{1} \mathrm{H}$ NMR $\left(600 \mathrm{MHz}, \mathrm{CDCl}_{3}\right): \delta=1.63\left(\mathrm{~s}, 3 \mathrm{H}, \mathrm{CH}_{3}\right), 3.22(\mathrm{~s}, 1 \mathrm{H}$, $\mathrm{OH}), 3.60$ (s, $3 \mathrm{H}, \mathrm{CO}_{2} \mathrm{CH}_{3}$ ), 3.87 (dd, $\left.J=12.9,4.5 \mathrm{~Hz}, 1 \mathrm{H}, \mathrm{CHAr}\right)$, $4.05(\mathrm{~d}, J=12.9 \mathrm{~Hz}, 1 \mathrm{H}, \mathrm{CHCO} \mathrm{Me}), 4.15(\mathrm{~d}, J=12.9,1.0 \mathrm{~Hz}, 1$ $\left.\mathrm{H}, \mathrm{CH}_{2}\right), 4.58\left(\mathrm{dd}, J=12.9,3.0 \mathrm{~Hz}, 1 \mathrm{H}, \mathrm{CH}_{2}\right), 4.78(\mathrm{~m}, 1 \mathrm{H}, \mathrm{CH}-$ $\left.\mathrm{NO}_{2}\right), 7.19-7.20\left(\mathrm{~m}, 2 \mathrm{H}, \mathrm{CH}_{\mathrm{Ar}}\right), 7.36\left(\mathrm{~s}, 1 \mathrm{H}, \mathrm{CH}_{\mathrm{Ar}}\right), 7.39-7.42(\mathrm{~m}$, $\left.1 \mathrm{H}, \mathrm{CH}_{\mathrm{Ar}}\right)$.

${ }^{13} \mathrm{C}$ NMR (151 MHz, $\left.\mathrm{CDCl}_{3}\right): \delta=28.6\left(\mathrm{CH}_{3}\right), 40.6(\mathrm{CHAr}), 47.9$ $\left(\mathrm{CO}_{2} \mathrm{CH}_{3}\right), 52.5\left(\mathrm{CHCO}_{2} \mathrm{CH}_{3}\right), 61.8\left(\mathrm{CH}_{2}\right), 84.4\left(\mathrm{CHNO}_{2}\right), 95.2$ $\left(\mathrm{C}_{\mathrm{q}} \mathrm{OH}\right), 122.9\left(\mathrm{C}_{\mathrm{Ar}}\right), 126.3\left(\mathrm{CH}_{\mathrm{Ar}}\right), 128.3\left(\mathrm{CH}_{\mathrm{Ar}}\right), 130.4\left(\mathrm{CH}_{\mathrm{Ar}}\right)$, $131.4\left(\mathrm{CH}_{\mathrm{Ar}}\right), 138.6\left(\mathrm{C}_{\mathrm{Ar}}\right), 172.3\left(\mathrm{CO}_{2} \mathrm{CH}_{3}\right)$.

Methyl (2S,3R,4R,5S)-2-Hydroxy-2-methyl-5-nitro-4-(thiophen-2-yl)tetrahydro-2H-pyran-3-carboxylate (trans-3g) Compound trans-3g was synthesized according to GP 1 ; yield: 178 mg (59\%); colorless solid; $\mathrm{mp} 111^{\circ} \mathrm{C}$ (benzene); $R_{f}=0.45$ ( $n$-pentane-Et $\left.{ }_{2} \mathrm{O}, 1: 1\right) ;[\alpha]_{\mathrm{D}}{ }^{20}-99.4\left(c 1.0, \mathrm{CHCl}_{3}\right) ;$ de $>95 \%$. The diastereomeric excess was determined by NMR spectroscopy.

IR (film): 3497 (s), 2315 (m), 2094 (m), 1734 (vs), 1547 (vs), 1439 (m), 1361 (vs), 1219 (m), 1135 (vs), 1060 (vs), $879(\mathrm{~m}), 840$ (m), $722 \mathrm{~cm}^{-1}$ (vs).

${ }^{1} \mathrm{H}$ NMR $\left(600 \mathrm{MHz}, \mathrm{CDCl}_{3}\right): \delta=1.49\left(\mathrm{~s}, 3 \mathrm{H}, \mathrm{CH}_{3}\right), 2.94(\mathrm{~d}$, $\left.J=11.9 \mathrm{~Hz}, 1 \mathrm{H}, \mathrm{CHCO}_{2} \mathrm{CH}_{3}\right), 3.49(\mathrm{~s}, 1 \mathrm{H}, \mathrm{OH}), 3.56(\mathrm{~s}, 3 \mathrm{H}$, $\left.\mathrm{CO}_{2} \mathrm{CH}_{3}\right), 4.09\left(\mathrm{dd}, J=10.9,5.0 \mathrm{~Hz}, 1 \mathrm{H}, \mathrm{CH}_{2}\right), 4.39(\mathrm{dd}, J=11.9$, $11.9 \mathrm{~Hz}, 1 \mathrm{H}, \mathrm{CHAr}$ ), 4.45 (dd, $\left.J=10.9,10.9 \mathrm{~Hz}, 1 \mathrm{H}, \mathrm{CH}_{2}\right), 4.85$ (ddd, $\left.J=11.4,10.9,5.0 \mathrm{~Hz}, 1 \mathrm{H}, \mathrm{CHNO}_{2}\right), 6.90-6.92(\mathrm{~m}, 2 \mathrm{H}$, $\left.\mathrm{CH}_{\mathrm{Ar}}\right), 7.20-7.23\left(\mathrm{~m}, 1 \mathrm{H}, \mathrm{CH}_{\mathrm{Ar}}\right)$.

${ }^{13} \mathrm{C} \mathrm{NMR}\left(151 \mathrm{MHz}, \mathrm{CDCl}_{3}\right): \delta=27.9\left(\mathrm{CH}_{3}\right), 39.3\left(\mathrm{CHCO}_{2} \mathrm{CH}_{3}\right)$, $52.6\left(\mathrm{CO}_{2} \mathrm{CH}_{3}\right), 56.4$ (CHAr), $61.5\left(\mathrm{CH}_{2}\right), 86.7\left(\mathrm{CHNO}_{2}\right), 94.9$ $\left(\mathrm{C}_{\mathrm{q}} \mathrm{OH}\right), 125.4\left(\mathrm{CH}_{\mathrm{Ar}}\right), 126.7\left(\mathrm{CH}_{\mathrm{Ar}}\right), 127.1\left(\mathrm{CH}_{\mathrm{Ar}}\right), 138.8\left(\mathrm{C}_{\mathrm{q}}\right)$, $171.5\left(\mathrm{CO}_{2} \mathrm{CH}_{3}\right)$.

MS (EI, $70 \mathrm{eV}): m / z(\%)=301\left([\mathrm{M}]^{+}, 11\right), 236(14), 211(27), 195$ (39), 179 (38) 177 (100), $168(23), 163$ (38), 151 (14), 137 (58), 123 (15), $111(11), 109$ (16), 97 (13), 65 (8).
Anal. Calcd for $\mathrm{C}_{12} \mathrm{H}_{15} \mathrm{NO}_{6} \mathrm{~S}: \mathrm{C}, 47.83 ; \mathrm{H}, 5.02 ; \mathrm{N}, 4.65$. Found: $\mathrm{C}$, $47.86 ; \mathrm{H}, 5.18 ; \mathrm{N}, 4.65$.

Methyl $(2 S, 3 R, 4 R, 5 R)-2-H y d r o x y-2-m e t h y l-5-n i t r o-4-(t h i o-$ phen-2-yl)tetrahydro-2H-pyran-3-carboxylate (cis-3g) Compound cis-3g was synthesized according to GP 1; yield: 102 mg (34\%); colorless solid; mp $63{ }^{\circ} \mathrm{C}$ (benzene); $[\alpha]_{\mathrm{D}}{ }^{20}-38.3$ (c 0.5 , $\mathrm{CHCl}_{3}$ ); de $>95 \% ; R_{f}=0.25$ ( $n$-pentane- $\left.\mathrm{Et}_{2} \mathrm{O}, 1: 1\right)$. The diastereomeric excess was determined by NMR spectroscopy.

${ }^{1} \mathrm{H}$ NMR $\left(600 \mathrm{MHz}, \mathrm{CDCl}_{3}\right): \delta=1.60\left(\mathrm{~s}, 3 \mathrm{H}, \mathrm{CH}_{3}\right), 3.37(\mathrm{~s}, 1 \mathrm{H}$, $\mathrm{OH}), 3.60\left(\mathrm{~s}, 3 \mathrm{H}, \mathrm{CO}_{2} \mathrm{CH}_{3}\right), 4.03(\mathrm{~d}, J=12.4 \mathrm{~Hz}, 1 \mathrm{H}, \mathrm{CH}-$ $\left.\mathrm{CO}_{2} \mathrm{CH}_{3}\right), 4.12\left(\mathrm{dd}, J=13.4,1.0 \mathrm{~Hz}, 1 \mathrm{H}, \mathrm{CH}_{2}\right), 4.14(\mathrm{dd}, J=12.4$, $4.5 \mathrm{~Hz}, 1 \mathrm{H}, \mathrm{CHAr}$ ), 4.56 (dd, $J=13.4,3.0 \mathrm{~Hz}, 1.0 \mathrm{~Hz}, 1 \mathrm{H}, \mathrm{CH}_{2}$ ), 4.83 (ddd, $\left.J=4.5,3.0,1.0 \mathrm{~Hz} 1 \mathrm{H}, \mathrm{CHNO}_{2}\right), 6.90-6.94(\mathrm{~m}, 2 \mathrm{H}$, $\left.\mathrm{CH}_{\mathrm{Ar}}\right), 7.18-7.20\left(\mathrm{~m}, 1 \mathrm{H}, \mathrm{CH}_{\mathrm{Ar}}\right)$.

${ }^{13} \mathrm{C} \mathrm{NMR}\left(151 \mathrm{MHz}, \mathrm{CDCl}_{3}\right): \delta=28.5\left(\mathrm{CH}_{3}\right), 37.0(\mathrm{CHAr}), 50.0$ $\left(\mathrm{CO}_{2} \mathrm{CH}_{3}\right), 52.5\left(\mathrm{CHCO}_{2} \mathrm{CH}_{3}\right), 61.6\left(\mathrm{CH}_{2}\right), 84.6\left(\mathrm{CHNO}_{2}\right), 95.2$ $\left(\mathrm{C}_{\mathrm{q}} \mathrm{OH}\right), 125.3\left(\mathrm{CH}_{\mathrm{Ar}}\right), 126.3\left(\mathrm{CH}_{\mathrm{Ar}}\right), 127.0\left(\mathrm{CH}_{\mathrm{Ar}}\right), 138.6\left(\mathrm{C}_{\mathrm{Ar}}\right)$, $172.5\left(\mathrm{CO}_{2} \mathrm{CH}_{3}\right)$.

Methyl $(2 S, 3 R, 4 S, 5 S)-4-(B e n z o[d][1,3]$ dioxol-5-yl)-2-hydroxy2-methyl-5-nitrotetrahydro-2H-pyran-3-carboxylate (trans-3h) Compound trans-3h was synthesized according to GP 1 ; yield: 221 mg $(65 \%)$; colorless solid; mp $166^{\circ} \mathrm{C}$ (benzene); $R_{f}=0.22(n$-pentane- $\left.\mathrm{Et}_{2} \mathrm{O}, 1: 1\right) ;[\alpha]_{\mathrm{D}}{ }^{20}-85.0$ (c $\left.1.0, \mathrm{CHCl}_{3}\right)$; de $>95 \%$. The diastereomeric excess was determined by NMR spectroscopy.

IR (film): 3503 (s), 3148 (s), 1733 (vs), 1552 (vs), 1493 (m), 1442 (vs), 1365 (vs), 1245 (vs), 1155 (vs), 1114 (vs), 1047 (vs), 933 (m), 886 (s), 807 (vs), $734 \mathrm{~cm}^{-1}$ (s).

${ }^{1} \mathrm{H}$ NMR $\left(400 \mathrm{MHz}, \mathrm{CDCl}_{3}\right): \delta=1.47\left(\mathrm{~s}, 3 \mathrm{H}, \mathrm{CH}_{3}\right), 2.88(\mathrm{~d}$, $\left.J=12.4 \mathrm{~Hz}, 1 \mathrm{H}, \mathrm{CHCO}_{2} \mathrm{CH}_{3}\right), 3.49(\mathrm{~s}, 1 \mathrm{H}, \mathrm{OH}), 3.50(\mathrm{~s}, 3 \mathrm{H}$, $\left.\mathrm{CO}_{2} \mathrm{CH}_{3}\right), 3.93(\mathrm{dd}, J=11.9,11.9 \mathrm{~Hz}, 1 \mathrm{H}, \mathrm{CHAr}), 4.08$ (dd, $\left.J=10.4,5.0 \mathrm{~Hz}, 1 \mathrm{H}, \mathrm{CH}_{2}\right), 4.45\left(\mathrm{dd}, J=10.9,10.4 \mathrm{~Hz}, 1 \mathrm{H}, \mathrm{CH}_{2}\right)$, 4.82 (ddd, $\left.J=11.9,10.9,5.0 \mathrm{~Hz}, 1 \mathrm{H}, \mathrm{CHNO}_{2}\right), 5.94(\mathrm{~m}, 2 \mathrm{H}$, $\left.\mathrm{OCH}_{2} \mathrm{O}\right), 6.65-6.72\left(\mathrm{~m}, 3 \mathrm{H}, \mathrm{CH}_{\mathrm{Ar}}\right)$.

${ }^{13} \mathrm{C} \mathrm{NMR}\left(100 \mathrm{MHz}, \mathrm{CDCl}_{3}\right): \delta=27.9\left(\mathrm{CH}_{3}\right), 43.5\left(\mathrm{CHCO}_{2} \mathrm{CH}_{3}\right)$, $52.5\left(\mathrm{CO}_{2} \mathrm{CH}_{3}\right), 55.3$ (CHAr), $61.5\left(\mathrm{CH}_{2}\right), 85.9\left(\mathrm{CHNO}_{2}\right), 94.8$ $\left(\mathrm{C}_{\mathrm{q}} \mathrm{OH}\right), 101.3\left(\mathrm{CH}_{2}\right), 107.8\left(\mathrm{CH}_{\mathrm{Ar}}\right), 108.6\left(\mathrm{CH}_{\mathrm{Ar}}\right), 121.5\left(\mathrm{CH}_{\mathrm{Ar}}\right)$, $129.4\left(\mathrm{C}_{\mathrm{q}}\right), 147.6\left(\mathrm{C}_{\mathrm{q}}\right), 148.0\left(\mathrm{C}_{\mathrm{q}}\right), 171.7\left(\mathrm{CO}_{2} \mathrm{CH}_{3}\right)$.

MS (CI, $70 \mathrm{eV}): m / z(\%)=339\left([\mathrm{M}]^{+}, 100\right), 249(17), 233(41), 215$ (84), 206 (41) 201 (31), 189 (11), 175 (74), 144 (21), 122 (12), 115 (20), 89 (12).

HRMS: $m / z$ calcd for $\mathrm{C}_{15} \mathrm{H}_{17} \mathrm{NO}_{8}+\mathrm{K}: 378.0591$; found: 378.0592 .

Methyl $(2 S, 3 R, 4 S, 5 R)-4-(B e n z o[d][1,3]$ dioxol-5-yl)-2-hydroxy2-methyl-5-nitrotetrahydro-2H-pyran-3-carboxylate (cis-3h)

Compound cis-3h was synthesized according to GP 1; yield: $88 \mathrm{mg}$ $(26 \%)$; colorless solid; mp $87^{\circ} \mathrm{C}(\mathrm{MeOH}) ; R_{f}=0.19$ (n-pentane$\left.\mathrm{Et}_{2} \mathrm{O}, 1: 2\right) ;[\alpha]_{\mathrm{D}}{ }^{20}-26.5\left(c 0.5, \mathrm{CHCl}_{3}\right) ; \mathrm{de}>95 \%$. The diastereomeric excess was determined by NMR spectroscopy.

${ }^{1} \mathrm{H} \mathrm{NMR}\left(600 \mathrm{MHz}, \mathrm{CDCl}_{3}\right): \delta=1.61\left(\mathrm{~s}, 3 \mathrm{H}, \mathrm{CH}_{3}\right), 3.33$ (br s, $1 \mathrm{H}$, $\mathrm{OH}), 3.59$ (s, $3 \mathrm{H}, \mathrm{CO}_{2} \mathrm{CH}_{3}$ ), 3.79 (dd, $J=12.9,4.0 \mathrm{~Hz}, 1 \mathrm{H}, \mathrm{CHAr}$ ), $3.99\left(\mathrm{~d}, J=12.9 \mathrm{~Hz}, 1 \mathrm{H}, \mathrm{CHCO}_{2} \mathrm{CH}_{3}\right), 4.11(\mathrm{~d}, J=13.4 \mathrm{~Hz}, 1 \mathrm{H}$, $\left.\mathrm{CH}_{2}\right), 4.54$ (dd, $\left.J=13.4,3.0 \mathrm{~Hz}, 1 \mathrm{H}, \mathrm{CH}_{2}\right), 4.75$ (dd, $J=4.0,3.0$ $\left.\mathrm{Hz}, 1 \mathrm{H}, \mathrm{CHNO}_{2}\right), 5.93\left(\mathrm{~d}, J=2 \mathrm{~Hz}, 2 \mathrm{H}, \mathrm{OCH}_{2} \mathrm{O}\right), 6.67-6.73(\mathrm{~m}$, $\left.3 \mathrm{H}, \mathrm{CH}_{\mathrm{Ar}}\right)$.

${ }^{13} \mathrm{C} \mathrm{NMR}\left(151 \mathrm{MHz}, \mathrm{CDCl}_{3}\right): \delta=28.6\left(\mathrm{CH}_{3}\right), 40.8(\mathrm{CHAr}), 52.4$ $\left(\mathrm{CO}_{2} \mathrm{CH}_{3}\right), 48.3\left(\mathrm{CHCO}_{2} \mathrm{CH}_{3}\right), 61.7\left(\mathrm{CH}_{2}\right), 84.9\left(\mathrm{CHNO}_{2}\right), 95.2$ $\left(\mathrm{C}_{\mathrm{q}} \mathrm{OH}\right), 101.2\left(\mathrm{CH}_{2}\right), 108.2\left(\mathrm{CH}_{\mathrm{Ar}}\right), 108.6\left(\mathrm{CH}_{\mathrm{Ar}}\right), 121.2\left(\mathrm{CH}_{\mathrm{Ar}}\right)$, $129.8\left(\mathrm{C}_{\mathrm{q}}\right), 147.4\left(\mathrm{C}_{\mathrm{q}}\right), 148.0\left(\mathrm{C}_{\mathrm{q}}\right), 172.8\left(\mathrm{CO}_{2} \mathrm{CH}_{3}\right)$.

\section{Methyl (4R)-6-Methyl-3-nitro-4-phenyl-3,4-dihydro-2H-pyran-} 5-carboxylate (4a)

Compound 4a was synthesized according to GP 2; yield: $222 \mathrm{mg}$ $(80 \%)$; colorless oil; $R_{f}=0.41\left(n\right.$-pentane-Et $\left.{ }_{2} \mathrm{O}, 6: 1\right) ;[\alpha]_{\mathrm{D}}{ }^{20}-171.3$ $\left(c 0.9, \mathrm{CHCl}_{3}\right)$; ee $($ trans $)=95 \%$; ee $($ cis $)=71 \%$; de $=71 \%$. The enantiomeric excess was determined by chiral stationary phase HPLC 
using a Chiralcel OD column ( $n$-heptane- $i$-PrOH, 7:3, flow rate 1.0 $\mathrm{mL} / \mathrm{min}, \lambda=254 \mathrm{~nm}$ ); $t_{\mathrm{R}}=7.38 \mathrm{~min}$ (minor), $t_{\mathrm{R}}=15.36 \mathrm{~min}(\mathrm{ma}-$ jor).

IR (film): 2945 (vs), 1708 (vs), 1627 (vs), 1552 (vs), 1449 (vs), 1377 (s), 1262 (vs), 1189 (w), 1097 (vs), 941 (m), 883 (m), 763 (s), $706 \mathrm{~cm}^{-1}$ (s).

${ }^{1} \mathrm{H}$ NMR (600 MHz, $\left.\mathrm{CDCl}_{3}\right): \delta=2.37\left(\mathrm{~s}, 3 \mathrm{H}, \mathrm{CH}_{3}\right), 3.53(\mathrm{~s}, 3 \mathrm{H}$, $\left.\mathrm{CO}_{2} \mathrm{CH}_{3}\right), 4.03\left(\mathrm{dd}, J=12.9,2.5 \mathrm{~Hz}, 1 \mathrm{H}, \mathrm{CH}_{2}\right), 4.64(\mathrm{dd}, J=2.5$, $\left.2.0 \mathrm{~Hz}, 1 \mathrm{H}, \mathrm{CHNO}_{2}\right), 4.69\left(\mathrm{dd}, J=12.9,2.0 \mathrm{~Hz}, 1 \mathrm{H}, \mathrm{CH}_{2}\right), 4.83$ (br s, $1 \mathrm{H}, \mathrm{CHPh}), 7.23-7.29\left(\mathrm{~m}, 3 \mathrm{H}, \mathrm{CH}_{\mathrm{Ar}}\right), 7.33-7.36(\mathrm{~m}, 2 \mathrm{H}$, $\mathrm{CH}_{\mathrm{Ar}}$ ).

${ }^{13} \mathrm{C}$ NMR $\left(151 \mathrm{MHz}, \mathrm{CDCl}_{3}\right): \delta=20.0\left(\mathrm{CH}_{3}\right), 39.9(\mathrm{CHPh}), 51.9$ $\left(\mathrm{CO}_{2} \mathrm{CH}_{3}\right), 61.8\left(\mathrm{CH}_{2}\right), 82.4\left(\mathrm{CHNO}_{2}\right), 100.4\left(\mathrm{C}_{\mathrm{q}}\right), 127.63\left(2 \mathrm{CH}_{\mathrm{Ar}}\right)$ $128.4\left(\mathrm{CH}_{\mathrm{Ar}}\right), 129.0\left(2 \mathrm{CH}_{\mathrm{Ar}}\right), 141.0\left(\mathrm{C}_{\mathrm{Ar}}\right), 165.2\left(\mathrm{C}_{\mathrm{q}} \mathrm{O}\right), 167.2$ $\left(\mathrm{CO}_{2} \mathrm{CH}_{3}\right)$.

MS (EI, $70 \mathrm{eV}): m / z(\%)=278\left([\mathrm{M}+1]^{+}, 27\right), 246(22), 230(100)$, 198 (48), 171 (17), 157 (14), 128 (13), 91 (13).

HRMS: $m / z$ calcd for $\mathrm{C}_{14} \mathrm{H}_{15} \mathrm{NO}_{5}+\mathrm{Na}$ : 300.0842 ; found: 300.0844 .

\section{Ethyl (4R)-6-Methyl-3-nitro-4-phenyl-3,4-dihydro-2H-pyran-} 5-carboxylate (4b)

Compound 4b was synthesized according to GP 2; yield: $248 \mathrm{mg}$ $(85 \%)$; colorless oil; $R_{f}=0.35$ (n-pentane-Et $\left.{ }_{2} \mathrm{O}, 8: 1\right) ;[\alpha]_{\mathrm{D}}{ }^{20}-266.9$ $\left(c 1.1, \mathrm{CHCl}_{3}\right)$; ee $($ trans $)=88 \%$; ee $($ cis $)=73 \%$; de $=64 \%$. The enantiomeric excess was determined by chiral stationary phase HPLC using a $(S, S)$ Whelk column ( $n$-heptane-EtOH, 97:3, flow rate 0.5 $\mathrm{mL} / \mathrm{min}, \lambda=254 \mathrm{~nm}$ ); $t_{\mathrm{R}}=12.54 \mathrm{~min}$ (major), $t_{\mathrm{R}}=13.68 \mathrm{~min}(\mathrm{mi}-$ nor), $t_{\mathrm{R}}=15.77 \min$ (major), $t_{\mathrm{R}}=18.46 \mathrm{~min}$ (minor).

IR (film): 2979 (m), 1701 (vs), 1625 (vs), 1550 (vs), 1451 (m), 1371 (s), 1253 (vs), 1154 (w), 1091 (vs), 942 (w), 899 (w), 844 (w), 761 (m), $703 \mathrm{~cm}^{-1}$ (s).

${ }^{1} \mathrm{H}$ NMR $\left(600 \mathrm{MHz}, \mathrm{CDCl}_{3}\right): \delta=0.98\left(\mathrm{t}, J=7.2 \mathrm{~Hz}, 3 \mathrm{H}, \mathrm{CH}_{2} \mathrm{CH}_{3}\right)$, 2.36 (s, $3 \mathrm{H}, \mathrm{CH}_{3}$ ), 3.90-4.10 (ddq, $J=12.5,7.2,2.3 \mathrm{~Hz}, 3 \mathrm{H}, \mathrm{CH}_{2}$ ), $4.60\left(\mathrm{dd}, J=2.3,2.3 \mathrm{~Hz}, 1 \mathrm{H}, \mathrm{CHNO}_{2}\right), 4.68(\mathrm{dd}, J=12.5,2.3 \mathrm{~Hz}$, $\left.1 \mathrm{H}, \mathrm{CH}_{2}\right), 4.81$ (br s, $\left.1 \mathrm{H}, \mathrm{CHPh}\right), 7.31-7.35\left(\mathrm{~m}, 2 \mathrm{H}, \mathrm{CH}_{\mathrm{Ar}}\right), 7.22$ $7.28\left(\mathrm{~m}, 3 \mathrm{H}, \mathrm{CH}_{\mathrm{Ar}}\right)$.

${ }^{13} \mathrm{C}$ NMR $\left(151 \mathrm{MHz}, \mathrm{CDCl}_{3}\right): \delta=13.9\left(\mathrm{CH}_{2} \mathrm{CH}_{3}\right), 19.8\left(\mathrm{CH}_{3}\right), 40.1$ $(\mathrm{CHPh}), 60.0\left(\mathrm{CH}_{2} \mathrm{CH}_{3}\right), 61.9\left(\mathrm{CH}_{2}\right), 82.6\left(\mathrm{CHNO}_{2}\right), 101.0\left(\mathrm{C}_{\mathrm{q}}\right)$, $127.6\left(\mathrm{CH}_{\mathrm{Ar}}\right), 127.8\left(2 \mathrm{CH}_{\mathrm{Ar}}\right), 129.0\left(2 \mathrm{CH}_{\mathrm{Ar}}\right), 141.3\left(\mathrm{C}_{\mathrm{Ar}}\right), 164.9$ $\left(\mathrm{C}_{\mathrm{q}} \mathrm{O}\right), 166.7\left(\mathrm{CO}_{2} \mathrm{CH}_{3}\right)$.

MS (EI, $70 \mathrm{eV}): m / z(\%)=292\left([\mathrm{M}+1]^{+}, 12\right), 244(100), 215(32)$, 198 (66), 171 (30), 157 (37), 128 (24), 115 (12), 91 (44).

HRMS: $m / z$ calcd for $\mathrm{C}_{15} \mathrm{H}_{17} \mathrm{NO}_{5}+\mathrm{Na}$ : 314.0999 ; found: 314.0999 .

Ethyl (4R)-3-Nitro-4,6-diphenyl-3,4-dihydro-2H-pyran-5-carboxylate (4c)

Compound $\mathbf{4 c}$ was synthesized according to GP 2; yield: $286 \mathrm{mg}$ (81\%); colorless oil; $R_{f}=0.39$ ( $n$-pentane- $\mathrm{Et}_{2} \mathrm{O} 6: 1$ ); $[\alpha]_{\mathrm{D}}{ }^{20}-267.8$ $\left(c 1.3, \mathrm{CHCl}_{3}\right)$; ee $($ trans $)=99 \%$; ee $($ cis $)=99 \%$; de $=98 \%$. The enantiomeric excess was determined by chiral stationary phase HPLC using a Chiralcel OD column ( $n$-heptane-EtOH, 9:1, flow rate 1.0 $\mathrm{mL} / \mathrm{min}, \lambda=254 \mathrm{~nm}$ ); $t_{\mathrm{R}}=10.44 \min \left(\right.$ minor), $t_{\mathrm{R}}=19.70 \mathrm{~min}(\mathrm{ma}-$ jor).

IR (film): 2317 (m), 2109 (m), 1888 (m), 1676 (vs), 1550 (vs), 1451 (s), 1357 (vs), 1290 (s), 1251 (s), $1212(\mathrm{~m}), 1150$ (m), 1098 (vs), 1052 (vs), 931 (m), $873(\mathrm{~m}), 762$ (s), $703 \mathrm{~cm}^{-1}$ (vs).

${ }^{1} \mathrm{H}$ NMR $\left(600 \mathrm{MHz}, \mathrm{CDCl}_{3}\right): \delta=0.75\left(\mathrm{t}, J=6.9 \mathrm{~Hz}, 3 \mathrm{H}, \mathrm{CH}_{3}\right)$, $3.78-3.89\left(\mathrm{~m}, 2 \mathrm{H}, \mathrm{CH}_{2} \mathrm{CH}_{3}\right), 4.25\left(\mathrm{dd}, J=12.4,2.0 \mathrm{~Hz}, 1 \mathrm{H}, \mathrm{CH}_{2}\right)$, $4.75\left(\mathrm{dd}, J=2.5,2.0 \mathrm{~Hz}, 1 \mathrm{H}, \mathrm{CHNO}_{2}\right), 4.86(\mathrm{dd}, J=12.4,2.5 \mathrm{~Hz}$, $\left.1 \mathrm{H}, \mathrm{CH}_{2}\right), 5.20$ (br s, $\left.1 \mathrm{H}, \mathrm{CHPh}\right), 7.24-7.27\left(\mathrm{~m}, 2 \mathrm{H}, \mathrm{CH}_{\mathrm{Ar}}\right), 7.29$ $7.49\left(\mathrm{~m}, 5 \mathrm{H}, \mathrm{C}_{6} \mathrm{H}_{5}\right)$.

${ }^{13} \mathrm{C} \mathrm{NMR}\left(151 \mathrm{MHz}, \mathrm{CDCl}_{3}\right): \delta=13.4\left(\mathrm{CH}_{3}\right), 40.8(\mathrm{CHPh}), 60.3$ $\left(\mathrm{CH}_{2}\right), 63.0\left(\mathrm{CH}_{2}\right), 82.9\left(\mathrm{CHNO}_{2}\right), 103.4\left(\mathrm{C}_{\mathrm{q}}\right), 127.9\left(2 \mathrm{CH}_{\mathrm{Ar}}\right)$ $128.1\left(2 \mathrm{CH}_{\mathrm{Ar}}\right), 128.6\left(\mathrm{CH}_{\mathrm{Ar}}\right), 129.1\left(2 \mathrm{CH}_{\mathrm{Ar}}\right), 129.3\left(2 \mathrm{CH}_{\mathrm{Ar}}\right), 129.8$ $\left(\mathrm{CH}_{\mathrm{Ar}}\right), 135.1\left(\mathrm{C}_{\mathrm{Ar}}\right), 140.5\left(\mathrm{C}_{\mathrm{Ar}}\right), 163.1\left(\mathrm{C}_{\mathrm{q}} \mathrm{O}\right), 166.9\left(\mathrm{CO}_{2} \mathrm{CH}_{3}\right)$.
MS (EI, $70 \mathrm{eV}): m / z(\%)=353\left([\mathrm{M}]^{+}, 3\right), 306(100), 277(12), 261$ (17), 233 (29), 204 (11), 128 (10), 105 (67), 91 (24), 77 (30).

HRMS: $m / z$ calcd for $\mathrm{C}_{20} \mathrm{H}_{19} \mathrm{NO}_{5}+\mathrm{Na}: 376.1155$; found: 376.1158 .

\section{1-[(4R)-6-Methyl-3-nitro-4-phenyl-3,4-dihydro-2H-pyran-5-} yl]ethanone (4d)

Compound 4d was synthesized according to GP 2; yield: $201 \mathrm{mg}$ (77\%); colorless oil; $R_{f}=0.40$ ( $n$-pentane- $\left.\mathrm{Et}_{2} \mathrm{O}, 8: 1\right) ;[\alpha]_{\mathrm{D}}{ }^{20}-298.7$ $\left(c 1.4, \mathrm{CHCl}_{3}\right)$; ee (trans) $=92 \%$; ee $($ cis $)=99 \%$; de $=87 \%$. The enantiomeric excess was determined by chiral stationary phase HPLC using a $(S, S)$ Whelk column ( $n$-heptane-EtOH, 97:3, flow rate 1.0 $\mathrm{mL} / \mathrm{min}, \lambda=254 \mathrm{~nm}) ; t_{\mathrm{R}}=13.12 \mathrm{~min}$ (major), $t_{\mathrm{R}}=18.59 \mathrm{~min}(\mathrm{mi}-$ nor), $t_{\mathrm{R}}=15.97 \mathrm{~min}$ (major), $t_{\mathrm{R}}=19.80 \mathrm{~min}$ (minor).

IR (film): 1689 (vs), 1622 (m), 1545 (vs), 1448 (m), 1353 (vs), 1277 (vs), 1122 (vs), 1081 (vs), 1021 (s), $916(\mathrm{w}), 846$ (m), 765 (vs), 696 $\mathrm{cm}^{-1}$ (vs).

${ }^{1} \mathrm{H}$ NMR $\left(600 \mathrm{MHz}, \mathrm{CDCl}_{3}\right): \delta=2.06\left(\mathrm{~s}, 3 \mathrm{H}, \mathrm{CH}_{3}\right), 2.31(\mathrm{~s}, 3 \mathrm{H}$, $\left.\mathrm{COCH}_{3}\right), 4.03\left(\mathrm{dd}, J=12.9,2.5 \mathrm{~Hz}, 1 \mathrm{H}, \mathrm{CH}_{2}\right), 4.64(\mathrm{dd}, J=2.5,2.0$ $\left.\mathrm{Hz}, 1 \mathrm{H}, \mathrm{CHNO}_{2}\right), 4.73\left(\mathrm{dd}, J=12.9,2.0 \mathrm{~Hz}, 1 \mathrm{H}, \mathrm{CH}_{2}\right), 4.83$ (br s, $1 \mathrm{H}, \mathrm{CHPh}), 7.24-7.27\left(\mathrm{~m}, 2 \mathrm{H}, \mathrm{CH}_{\mathrm{Ar}}\right), 7.29-7.32\left(\mathrm{~m}, 1 \mathrm{H}, \mathrm{CH}_{\mathrm{Ar}}\right)$, $7.37-7.41\left(\mathrm{~m}, 2 \mathrm{H}, \mathrm{CH}_{\mathrm{Ar}}\right)$.

${ }^{13} \mathrm{C}$ NMR $\left(151 \mathrm{MHz}, \mathrm{CDCl}_{3}\right): \delta=20.5\left(\mathrm{CH}_{3}\right), 29.2\left(\mathrm{COCH}_{3}\right), 40.7$ $(\mathrm{CHPh}), 61.7\left(\mathrm{CH}_{2}\right), 82.5\left(\mathrm{CHNO}_{2}\right), 109.1\left(\mathrm{C}_{\mathrm{q}}\right), 128.2\left(2 \mathrm{CH}_{\mathrm{Ar}}\right)$, $128.2\left(\mathrm{CH}_{\mathrm{Ar}}\right), 129.5\left(2 \mathrm{CH}_{\mathrm{Ar}}\right), 140.3\left(\mathrm{C}_{\mathrm{Ar}}\right), 164.1\left(\mathrm{C}_{\mathrm{q}} \mathrm{O}\right), 198.2(\mathrm{CO}-$ $\mathrm{CH}_{3}$ ).

MS (EI, $70 \mathrm{eV}): m / z(\%)=261\left([\mathrm{M}]^{+}, 8\right), 215(100), 199(46), 171$ (58), 155 (27), 128 (32), 115 (19), 91 (68), 77 (15), 59 (22).

HRMS: $m / z$ calcd for $\mathrm{C}_{14} \mathrm{H}_{16} \mathrm{NO}_{4}$ : 262.1074; found: 262.1073 .

\section{Methyl (4R)-6-Methyl-3-nitro-4-(p-tolyl)-3,4-dihydro-2H- pyran-5-carboxylate (4e)}

Compound 4e was synthesized according to GP 2; yield: $236 \mathrm{mg}$ $(81 \%)$; colorless oil; $R_{f}=0.34$ ( $n$-pentane-Et $\left.2 \mathrm{O}, 8: 1\right) ;[\alpha]_{\mathrm{D}}{ }^{20}-226.5$ $\left(\right.$ c $\left.0.9, \mathrm{CHCl}_{3}\right)$; ee $($ trans $)=88 \%$; ee $($ cis $)=89 \%$; de $=45 \%$. The enantiomeric excess was determined by chiral stationary phase HPLC using a $(S, S)$ Whelk column ( $n$-heptane-EtOH, 97:3, flow rate 0.5 $\mathrm{mL} / \mathrm{min}, \lambda=254 \mathrm{~nm}$ ); $t_{\mathrm{R}}=13.00 \mathrm{~min}$ (major), $t_{\mathrm{R}}=14.78 \mathrm{~min}(\mathrm{mi}-$ nor), $t_{\mathrm{R}}=16.63 \mathrm{~min}$ (major), $t_{\mathrm{R}}=20.29 \mathrm{~min}$ (minor).

IR (film): 2940 (vs), 1704 (vs), 1624 (vs), 1549 (vs), 1441 (s), 1362 (s), 1256 (vs), 1191 (w), 1095 (vs), 936 (m), 881 (m), 800 (m), 724 $\mathrm{cm}^{-1}(\mathrm{~m})$.

${ }^{1} \mathrm{H}$ NMR $\left(600 \mathrm{MHz}, \mathrm{CDCl}_{3}\right): \delta=2.30\left(\mathrm{~s}, 3 \mathrm{H}, \mathrm{CH}_{3}\right), 2.34(\mathrm{~s}, 3 \mathrm{H}$, $\left.\mathrm{CH}_{3}\right), 3.55$ (s, $3 \mathrm{H}, \mathrm{CO}_{2} \mathrm{CH}_{3}$ ), 4.04 (dd, $J=12.9,2.0 \mathrm{~Hz}, 1 \mathrm{H}, \mathrm{CH}_{2}$ ), $4.58\left(\mathrm{dd}, J=2.0,2.0 \mathrm{~Hz}, 1 \mathrm{H}, \mathrm{CHNO}_{2}\right), 4.69(\mathrm{dd}, J=12.9,2.0 \mathrm{~Hz}$, $\left.1 \mathrm{H}, \mathrm{CH}_{2}\right), 4.79$ (br s, $\left.1 \mathrm{H}, \mathrm{CHAr}\right), 7.04-7.08\left(\mathrm{~m}, 4 \mathrm{H}, \mathrm{CH}_{\mathrm{Ar}}\right.$ ).

${ }^{13} \mathrm{C}$ NMR $\left(151 \mathrm{MHz}, \mathrm{CDCl}_{3}\right): \delta=19.8\left(\mathrm{CH}_{3}\right), 21.0\left(\mathrm{CH}_{3}\right), 39.5$ (CHAr), $51.5\left(\mathrm{CO}_{2} \mathrm{CH}_{3}\right), 61.8\left(\mathrm{CH}_{2}\right), 82.6\left(\mathrm{CHNO}_{2}\right), 100.6\left(\mathrm{C}_{\mathrm{q}}\right)$, $127.7\left(2 \mathrm{CH}_{\mathrm{Ar}}\right), 129.8\left(2 \mathrm{CH}_{\mathrm{Ar}}\right), 137.4\left(\mathrm{C}_{\mathrm{Ar}}\right), 138.0\left(\mathrm{C}_{\mathrm{Ar}}\right), 165.0$ $\left(\mathrm{C}_{\mathrm{q}} \mathrm{O}\right), 167.4\left(\mathrm{CO}_{2} \mathrm{CH}_{3}\right)$.

MS (EI, $70 \mathrm{eV}): m / z(\%)=291\left([\mathrm{M}]^{+}, 15\right), 244(100), 229(36), 213$ (80), 171 (47), 143 (19), 128 (25), 115 (19), 105 (63), 91 (10).

HRMS: $m / z$ calcd for $\mathrm{C}_{15} \mathrm{H}_{17} \mathrm{NO}_{5}+\mathrm{Na}: 314.0999$; found: 314.0999 .

\section{Methyl (4R)-4-(3-Bromophenyl)-6-methyl-3-nitro-3,4-dihydro- $2 \mathrm{H}$-pyran-5-carboxylate (4f)}

Compound $\mathbf{4 f}$ was synthesized according to GP 2; yield: $306 \mathrm{mg}$ $(86 \%)$; colorless oil; $R_{f}=0.26\left(n\right.$-pentane- $\left.\mathrm{Et}_{2} \mathrm{O}, 8: 1\right) ;[\alpha]_{\mathrm{D}}{ }^{20}-271.3$ $\left(c 1.1, \mathrm{CHCl}_{3}\right)$; ee (trans) $=88 \%$; ee $($ cis $)=78 \%$; de $=76 \%$. The enantiomeric excess was determined by chiral stationary phase HPLC using a Chiralcel OJ column ( $n$-heptane-EtOH, 80:20, flow rate 1.0 $\mathrm{mL} / \mathrm{min}, \lambda=254 \mathrm{~nm}$ ); $t_{\mathrm{R}}=4.68 \mathrm{~min}$ (major), $t_{\mathrm{R}}=7.69 \min$ (minor), $t_{\mathrm{R}}=13.50 \mathrm{~min}$ (major), $t_{\mathrm{R}}=20.80 \mathrm{~min}$ (minor).

IR (film): 2951 (vs), 1710 (vs), 1626 (vs), 1555 (vs), 1435 (vs), 1382 (vs), 1359 (vs), 1259 (vs), 1189 (s), 1150 (m), 1102 (vs), 1051 (s), $941(\mathrm{~s}), 890(\mathrm{~s}), 784(\mathrm{~s}), 699 \mathrm{~cm}^{-1}(\mathrm{~s})$. 
${ }^{1} \mathrm{H}$ NMR (400 MHz, $\left.\mathrm{CDCl}_{3}\right): \delta=2.35\left(\mathrm{~s}, 3 \mathrm{H}, \mathrm{CH}_{3}\right), 3.53(\mathrm{~s}, 3 \mathrm{H}$, $\left.\mathrm{CO}_{2} \mathrm{CH}_{3}\right), 3.99\left(\mathrm{dd}, J=12.8,2.0 \mathrm{~Hz}, 1 \mathrm{H}, \mathrm{CH}_{2}\right), 4.57(\mathrm{dd}, J=2.0$, $2.0 \mathrm{~Hz}, 1 \mathrm{H}, \mathrm{CHNO}_{2}$ ), 4.69 (dd, $\left.J=12.8,2.0 \mathrm{~Hz}, 1 \mathrm{H}, \mathrm{CH}_{2}\right), 4.79$ (br s, $1 \mathrm{H}, \mathrm{CHAr}), 7.10-7.24\left(\mathrm{~m}, 3 \mathrm{H}, \mathrm{CH}_{\mathrm{Ar}}\right), 7.36-7.41(\mathrm{~m}, 1 \mathrm{H}$, $\mathrm{CH}_{\mathrm{Ar}}$ ).

${ }^{13} \mathrm{C}$ NMR $\left(100 \mathrm{MHz}, \mathrm{CDCl}_{3}\right): \delta=20.1\left(\mathrm{CH}_{3}\right), 39.6(\mathrm{CHAr}), 51.5$ $\left(\mathrm{CO}_{2} \mathrm{CH}_{3}\right), 61.8\left(\mathrm{CH}_{2}\right), 82.0\left(\mathrm{CHNO}_{2}\right), 99.8\left(\mathrm{C}_{\mathrm{q}}\right), 123.3\left(\mathrm{C}_{\mathrm{Ar}}\right), 126.5$ $\left(\mathrm{CH}_{\mathrm{Ar}}\right), 130.7\left(\mathrm{CH}_{\mathrm{Ar}}\right), 130.9\left(\mathrm{CH}_{\mathrm{Ar}}\right), 131.0\left(\mathrm{CH}_{\mathrm{Ar}}\right), 143.5\left(\mathrm{C}_{\mathrm{Ar}}\right)$, $165.8\left(\mathrm{C}_{\mathrm{q}} \mathrm{O}\right), 166.9\left(\mathrm{CO}_{2} \mathrm{CH}_{3}\right)$.

MS (EI, $70 \mathrm{eV}): m / z(\%)=355\left([\mathrm{M}]^{+}, 8\right), 324(11), 310(100), 276$ (33), 251 (20), 235 (18), 229 (28), 197 (25), 169 (31), 155 (18), 128 (33), 115 (15).

HRMS: $m / z$ calcd for $\mathrm{C}_{14} \mathrm{H}_{14} \mathrm{BrNO}_{5}+\mathrm{Na}$ : 377.9948; found: 377.9951 .

\section{Methyl (4R)-6-Methyl-3-nitro-4-(thiophen-2-yl)-3,4-dihydro-} 2H-pyran-5-carboxylate (4g)

Compound 4g was synthesized according to GP 2; yield: $232 \mathrm{mg}$ (82\%); colorless oil; $R_{f}=0.23$ ( $n$-pentane- $\left.\mathrm{Et}_{2} \mathrm{O}, 8: 1\right) ;[\alpha]_{\mathrm{D}}{ }^{20}-384.3$ $\left(c 1.3, \mathrm{CHCl}_{3}\right)$; ee $($ trans $)=90 \%$; ee $($ cis $)=94 \%$; de $=26 \%$. The enantiomeric excess was determined by chiral stationary phase HPLC using a Chiralpak IA column ( $n$-heptane-EtOH, 95:5, flow rate 0.7 $\mathrm{mL} / \mathrm{min}, \lambda=254 \mathrm{~nm}$ ); $t_{\mathrm{R}}=13.64 \mathrm{~min}$ (minor), $t_{\mathrm{R}}=15.20 \mathrm{~min}$ (major), $t_{\mathrm{R}}=14.63 \mathrm{~min}$ (major), $t_{\mathrm{R}}=16.40 \mathrm{~min}$ (minor).

IR (film): 2950 (vs), 1704 (vs), 1621 (vs), 1551 (vs), 1441 (s), 1361 (s), 1258 (vs), 1203 (s), 1094 (vs), 933 (m), 883 (m), 845 (m), 789 (w), $706 \mathrm{~cm}^{-1}$ (vs).

${ }^{1} \mathrm{H}$ NMR $\left(600 \mathrm{MHz}, \mathrm{CDCl}_{3}\right): \delta=2.33\left(\mathrm{~s}, 3 \mathrm{H}, \mathrm{CH}_{3}\right), 3.61(\mathrm{~s}, 3 \mathrm{H}$, $\left.\mathrm{CO}_{2} \mathrm{CH}_{3}\right), 4.20\left(\mathrm{dd}, J=12.9,2.0 \mathrm{~Hz}, 1 \mathrm{H}, \mathrm{CH}_{2}\right), 4.70(\mathrm{dd}, J=2.0$, $\left.2.0 \mathrm{~Hz}, 1 \mathrm{H}, \mathrm{CHNO}_{2}\right), 4.88\left(\mathrm{dd}, J=12.9,2.0 \mathrm{~Hz}, 1 \mathrm{H}, \mathrm{CH}_{2}\right), 5.05$ (br s, $1 \mathrm{H}, \mathrm{CHAr}), 6.88\left(\mathrm{~m}, 1 \mathrm{H}, \mathrm{CH}_{\mathrm{Ar}}\right), 6.95\left(\mathrm{~m}, 1 \mathrm{H}, \mathrm{CH}_{\mathrm{Ar}}\right), 7.24$ $\left(\mathrm{m}, 1 \mathrm{H}, \mathrm{CH}_{\mathrm{Ar}}\right)$.

${ }^{13} \mathrm{C}$ NMR $\left(151 \mathrm{MHz}, \mathrm{CDCl}_{3}\right): \delta=20.0\left(\mathrm{CH}_{3}\right), 34.9(\mathrm{CHPh}), 51.5$ $\left(\mathrm{CO}_{2} \mathrm{CH}_{3}\right), 62.3\left(\mathrm{CH}_{2}\right), 81.9\left(\mathrm{CHNO}_{2}\right), 101.4\left(\mathrm{C}_{\mathrm{q}}\right), 125.31\left(\mathrm{CH}_{\mathrm{Ar}}\right)$, $125.9\left(\mathrm{CH}_{\mathrm{Ar}}\right), 127.4\left(\mathrm{CH}_{\mathrm{Ar}}\right), 144.9\left(\mathrm{C}_{\mathrm{Ar}}\right), 165.0\left(\mathrm{C}_{\mathrm{q}} \mathrm{O}\right), 167.0$ $\left(\mathrm{CO}_{2} \mathrm{CH}_{3}\right)$.

MS (EI, $70 \mathrm{eV}): m / z(\%)=283\left([\mathrm{M}]^{+}, 11\right), 236(69), 205(100), 176$ (16), 163 (72), 135 (21), 97 (57), 91 (13)

HRMS: $m / z$ calcd for $\mathrm{C}_{12} \mathrm{H}_{13} \mathrm{NO}_{5} \mathrm{~S}+\mathrm{Na}$ : 306.0407; found: 306.0409 .

\section{Methyl (4R)-4-(Benzo[d][1,3]dioxol-5-yl)-6-methyl-3-nitro 3,4-} dihydro-2H-pyran-5-carboxylate (4h)

Compound $\mathbf{4 h}$ was synthesized according to GP 2; yield: $260 \mathrm{mg}$ (81\%); colorless oil; $R_{f}=0.36\left(n\right.$-pentane- $\left.\mathrm{Et}_{2} \mathrm{O}, 6: 1\right) ;[\alpha]_{\mathrm{D}}{ }^{20}-311.8$ (c $\left.1.2, \mathrm{CHCl}_{3}\right)$; ee $($ trans $)=91 \%$; ee $($ cis $)=83 \%$; de $=43 \%$. The enantiomeric excess was determined by chiral stationary phase HPLC using a $(S, S)$ Whelk column ( $n$-heptane-EtOH, 97:3, flow rate 1.0 $\mathrm{mL} / \mathrm{min}, \lambda=254 \mathrm{~nm}$ ); $t_{\mathrm{R}}=12.36 \mathrm{~min}$ (major), $t_{\mathrm{R}}=15.20 \mathrm{~min}(\mathrm{mi}-$ nor), $t_{\mathrm{R}}=17.75 \mathrm{~min}$ (major), $t_{\mathrm{R}}=23.79 \mathrm{~min}$ (minor).

IR (film): 2952 (vs), 1713 (vs), 1633 (vs), 1555 (vs), 1488 (vs), 1446 (vs), 1383 (vs), 1235 (s), 1189 (m), 1036 (vs), 930 (vs), 872 (vs), $818(\mathrm{~s}), 762(\mathrm{~m}), 735(\mathrm{~s}), 762 \mathrm{~cm}^{-1}(\mathrm{~m})$.

${ }^{1} \mathrm{H}$ NMR (600 MHz, $\left.\mathrm{CDCl}_{3}\right): \delta=2.34\left(\mathrm{~s}, 3 \mathrm{H}, \mathrm{CH}_{3}\right), 3.57(\mathrm{~s}, 3 \mathrm{H}$, $\left.\mathrm{CO}_{2} \mathrm{CH}_{3}\right), 4.01\left(\mathrm{dd}, J=12.4,2.0 \mathrm{~Hz}, 1 \mathrm{H}, \mathrm{CH}_{2}\right), 4.52(\mathrm{dd}, J=2.0$, $\left.2.0 \mathrm{~Hz}, 1 \mathrm{H}, \mathrm{CHNO}_{2}\right), 4.64\left(\mathrm{dd}, J=12.4,12.4 \mathrm{~Hz}, 1 \mathrm{H}, \mathrm{CH}_{2}\right), 4.73$ (br s, $1 \mathrm{H}, \mathrm{CHAr}), 5.94\left(\mathrm{~s}, 2 \mathrm{H}, \mathrm{CH}_{2}\right), 6.60-6.69\left(\mathrm{~m}, 3 \mathrm{H}, \mathrm{CH}_{\mathrm{Ar}}\right)$.

${ }^{13} \mathrm{C}$ NMR (151 MHz, $\left.\mathrm{CDCl}_{3}\right): \delta=20.0\left(\mathrm{CH}_{3}\right), 39.6$ (CHAr), 51.5 $\left(\mathrm{CO}_{2} \mathrm{CH}_{3}\right), 61.9\left(\mathrm{CH}_{2}\right), 82.5\left(\mathrm{CHNO}_{2}\right), 100.7\left(\mathrm{C}_{\mathrm{q}}\right), 101.3\left(\mathrm{CH}_{2}\right)$, 108.2 $\left(\mathrm{CH}_{\mathrm{Ar}}\right), 108.7\left(\mathrm{CH}_{\mathrm{Ar}}\right), 121.1\left(\mathrm{CH}_{\mathrm{Ar}}\right), 134.9\left(\mathrm{C}_{\mathrm{Ar}}\right), 147.1\left(\mathrm{C}_{\mathrm{Ar}}\right)$, $148.3\left(\mathrm{C}_{\mathrm{Ar}}\right), 165.0\left(\mathrm{C}_{\mathrm{q}} \mathrm{O}\right), 167.2\left(\mathrm{CO}_{2} \mathrm{CH}_{3}\right)$.

MS (EI, $70 \mathrm{eV}): m / z(\%)=321\left([\mathrm{M}]^{+}, 50\right), 274(23), 243(100), 214$ (28), 201 (57), 173 (11), 143 (10), 135 (68), 115 (26), 77 (12).
HRMS: $m / z$ calcd for $\mathrm{C}_{15} \mathrm{H}_{15} \mathrm{NO}_{7}+\mathrm{Na}$ : 344.0741; found: 344.0744 .

\section{Methyl (2S,3R,4S,5S)-2-Methoxy-2-methyl-5-nitro-4-phen-} yltetrahydro-2H-pyran-3-carboxylate (7)

In a glass vial equipped with a magnetic stirring bar, trans-3a (295 $\mathrm{mg}, 1 \mathrm{mmol}$ ) was added to a mixture of PTSA (38 mg, $20 \mathrm{~mol} \%$ ) in $\mathrm{MeOH}(6 \mathrm{~mL})$ and stirred for $8 \mathrm{~h}$ at r.t. The reaction was monitored by TLC. After completion, the solvent was evaporated and the crude reaction mixture was purified by column chromatography (silica gel, $n$-pentane- $\left.\mathrm{Et}_{2} \mathrm{O}, 10: 1\right)$ to yield 7 in $257 \mathrm{mg}(62 \%)$ as a colorless solid; $\mathrm{mp} 83{ }^{\circ} \mathrm{C} ; R_{f}=0.40\left(n\right.$-pentane- $\left.\mathrm{Et}_{2} \mathrm{O}, 4: 1\right) ;[\alpha]_{\mathrm{D}}{ }^{20}$ -71.3 (c 1.0, $\left.\mathrm{CHCl}_{3}\right)$; ee $=99 \%$; de $=98 \%$. The enantiomeric excess was determined by chiral stationary phase HPLC using a Chiralcel OD column ( $n$-heptane- $i$ - $\mathrm{PrOH}, 97: 3$, flow rate $0.7 \mathrm{~mL} / \mathrm{min}$, $\lambda=254 \mathrm{~nm}$ ); $t_{\mathrm{R}}=11.45 \mathrm{~min}$ (minor), $t_{\mathrm{R}}=14.89 \mathrm{~min}$ (major).

IR (film): 1731 (vs), 1548 (vs), 1454 (m), 1386 (m), 1267 (vs), 1173 (vs), 1118 (s), 1039 (vs), 869 (s), 760 (vs), $699 \mathrm{~cm}^{-1}$ (vs).

${ }^{1} \mathrm{H}$ NMR $\left(600 \mathrm{MHz}, \mathrm{CDCl}_{3}\right): \delta=1.51\left(\mathrm{~s}, 3 \mathrm{H}, \mathrm{CH}_{3}\right), 2.99(\mathrm{~d}$, $\left.J=12.4 \mathrm{~Hz}, 1 \mathrm{H}, \mathrm{CHCO}_{2} \mathrm{CH}_{3}\right), 3.33\left(\mathrm{~s}, 3 \mathrm{H}, \mathrm{OCH}_{3}\right), 3.47(\mathrm{~s}, 3 \mathrm{H}$, $\left.\mathrm{CO}_{2} \mathrm{CH}_{3}\right), 4.04$, (dd, $\left.J=10.9,5.5 \mathrm{~Hz}, 1 \mathrm{H}, \mathrm{CH}_{2}\right), 4.08(\mathrm{dd}, J=10.9$, $10.4 \mathrm{~Hz}, 1 \mathrm{H}, \mathrm{CH}_{2}$ ), 4.16 (dd, $J=11.9,11.9 \mathrm{~Hz}, 1 \mathrm{H}, \mathrm{CHPh}$ ), 4.83 (ddd, $\left.J=11.9,10.4,5.5 \mathrm{~Hz}, 1 \mathrm{H}, \mathrm{CHNO}_{2}\right), 7.21-7.29(\mathrm{~m}, 5 \mathrm{H}$, $\mathrm{C}_{6} \mathrm{H}_{5}$ )

${ }^{13} \mathrm{C} \mathrm{NMR}\left(151 \mathrm{MHz}, \mathrm{CDCl}_{3}\right): \delta=22.1\left(\mathrm{CH}_{3}\right), 42.6\left(\mathrm{CHCO}_{2} \mathrm{CH}_{3}\right)$, $48.7\left(\mathrm{COCH}_{3}\right), 51.9\left(\mathrm{CO}_{2} \mathrm{CH}_{3}\right), 56.3(\mathrm{CHPh}), 61.2\left(\mathrm{CH}_{2}\right), 86.5$ $\left(\mathrm{CHNO}_{2}\right), 98.2\left(\mathrm{C}_{\mathrm{q}} \mathrm{OMe}\right), 128.0\left(2 \mathrm{CH}_{\mathrm{Ar}}\right), 128.1\left(\mathrm{CH}_{\mathrm{Ar}}\right), 128.8(2$ $\left.\mathrm{CH}_{\mathrm{Ar}}\right), 136.7\left(\mathrm{C}_{\mathrm{q}}\right), 198.7\left(\mathrm{CO}_{2} \mathrm{CH}_{3}\right)$.

MS (EI, $70 \mathrm{eV}): m / z(\%)=309\left([\mathrm{M}]^{+}, 1\right), 128(29), 248(11), 235$ (14), 188 (100), 173 (15), 157 (26), 129 (87), 117 (62), 103 (28), 91 (19), 77 (10), 59 (19).

HRMS: $m / z$ calcd for $\mathrm{C}_{15} \mathrm{H}_{19} \mathrm{NO}_{6}+\mathrm{Na}$ : 332.1105 ; found: 332.1104 .

\section{Methyl $(2 S, 3 R, 4 S, 5 S)$-2-Methyl-5-nitro-4-phenyltetrahydro-}

\section{H-pyran-3-carboxylate (8)}

In a glass vial equipped with a magnetic stirring bar, trans-3a (295 $\mathrm{mg}, 1 \mathrm{mmol})$ was added to a mixture $\mathrm{Et}_{3} \mathrm{SiH}(240 \mu \mathrm{L}, 1.5 \mathrm{mmol})$, trifluoroborane diethyl etherate $(130 \mu \mathrm{L}, 1.0 \mathrm{mmol})$ in $\mathrm{CH}_{2} \mathrm{Cl}_{2}(6$ $\mathrm{mL}$ ) and stirred for $6 \mathrm{~h}$ at $0{ }^{\circ} \mathrm{C}$. The reaction was monitored by TLC. The reaction mixture was warmed up to r.t. and quenched with sat. aq $\mathrm{NaHCO}_{3}(5 \mathrm{~mL})$, the organic phase was separated, and the aqueous phase was extracted with EtOAc $(3 \times 5 \mathrm{~mL})$. The organic extracts were combined, dried $\left(\mathrm{Na}_{2} \mathrm{SO}_{4}\right)$, and concentrated under reduced pressure. The residue was purified by chromatography (silica gel, $n$-pentane-EtOAc, 6:1) to yield 8 in $211 \mathrm{mg}$ (72\%); mp $131{ }^{\circ} \mathrm{C} ; R_{f}=0.33\left(n\right.$-pentane- $\left.\mathrm{Et}_{2} \mathrm{O}, 4: 1\right) ;[\alpha]_{\mathrm{D}}{ }^{20}-64.4$ (c 1.0, CH$\left.\mathrm{Cl}_{3}\right)$; ee $=98 \%$; de $=97 \%$. The enantiomeric excess was determined by chiral stationary phase HPLC using a Chiralcel OJ column $(n-$ heptane-EtOH, 7:3, flow rate $0.7 \mathrm{~mL} / \mathrm{min}, \lambda=254 \mathrm{~nm}) ; t_{\mathrm{R}}=9.35$ $\min$ (minor), $t_{\mathrm{R}}=12.50 \mathrm{~min}$ (major).

IR (film): 1729 (vs), 1542 (vs), 1450 (s), 1344 (vs), 1270 (m), 1196 (s), 1121 (vs), 1077 (vs), 903 (m), 767 (vs), $701 \mathrm{~cm}^{-1}$ (vs).

${ }^{1} \mathrm{H}$ NMR $\left(400 \mathrm{MHz}, \mathrm{CDCl}_{3}\right): \delta=1.24\left(\mathrm{~d}, J=6.0 \mathrm{~Hz}, 3 \mathrm{H}, \mathrm{CH}_{3}\right)$, 2.65 (dd, $\left.J=11.9,9.9 \mathrm{~Hz}, 1 \mathrm{H}, \mathrm{CHCO}_{2} \mathrm{CH}_{3}\right), 3.43(\mathrm{~s}, 3 \mathrm{H}$, $\left.\mathrm{CO}_{2} \mathrm{CH}_{3}\right), 3.75(\mathrm{dd}, J=11.9,11.4 \mathrm{~Hz}, 1 \mathrm{H}, \mathrm{CHPh}), 3.82(\mathrm{~m}, 1 \mathrm{H}$, $\left.\mathrm{CHCH}_{3}\right), 3.91\left(\mathrm{dd}, J=10.9,10.4 \mathrm{~Hz}, 1 \mathrm{H}, \mathrm{CH}_{2}\right), 4.41(\mathrm{dd}, J=10.4$, $4.5 \mathrm{~Hz}, 1 \mathrm{H}, \mathrm{CH}_{2}$ ), 4.90 (ddd, $J=11.4,10.94 .5 \mathrm{~Hz}, 1 \mathrm{H}, \mathrm{CHNO}_{2}$ ), 7.19-7.32 (m, $\left.5 \mathrm{H}, \mathrm{C}_{6} \mathrm{H}_{5}\right)$.

${ }^{13} \mathrm{C}$ NMR $\left(100 \mathrm{MHz}, \mathrm{CDCl}_{3}\right): \delta=19.5\left(\mathrm{CH}_{3}\right), 48.1\left(\mathrm{CHCO}_{2} \mathrm{CH}_{3}\right)$, $51.9\left(\mathrm{CO}_{2} \mathrm{CH}_{3}\right), 55.3(\mathrm{CHPh}), 69.2\left(\mathrm{CH}_{2}\right), 75.7\left(\mathrm{CHCH}_{3}\right), 85.8$ $\left(\mathrm{CHNO}_{2}\right), 127.6\left(2 \mathrm{CH}_{\mathrm{Ar}}\right), 128.4\left(\mathrm{CH}_{\mathrm{Ar}}\right), 129.0\left(2 \mathrm{CH}_{\mathrm{Ar}}\right), 135.9$ $\left(\mathrm{C}_{\mathrm{q}}\right), 170.8\left(\mathrm{CO}_{2} \mathrm{CH}_{3}\right)$.

MS (EI, $70 \mathrm{eV}): m / z(\%)=279\left([\mathrm{M}]^{+}, 15\right), 232(25), 189(34), 172$ (100), 157 (73), 129 (63), 115 (31), 103 (18), 91 (27), 77 (15), 59 (22).

HRMS: $m / z$ calcd for $\mathrm{C}_{14} \mathrm{H}_{17} \mathrm{NO}_{5}+\mathrm{Na}: 302.1004$; found: 302.1003 . 


\section{Acknowledgment}

D. E. thanks the European Research Council for an ERC Advanced Grant (DOMINOCAT). We thank the BASE SE for donation of chemicals.

Supporting Information for this article is available online at http://www.thieme-connect.com/ejournals/toc/synthesis.

\section{References}

(1) For reviews on organocatalytic domino reactions, see: (a) Sheffler, U.; Mahrwald, R. Chem. Eur. J. 2013, 19, 14346. (b) Bonne, D.; Constanieux, T.; Coquerel, Y.; Rodriguez, J. Chem. Eur. J. 2013, 19, 2218. (c) Pellissier, H. Chem. Rev. 2013, 113, 442. (d) Grossmann, A.; Enders, D. Angew. Chem. Int. Ed. 2011, 51, 314. (e) Enders, D.; Jeanty, M.; Grondal, C. Nat. Chem. 2010, 2, 167. (f) Alba, A.-N.; Companyo, X.; Viciano, M.; Rios, R. Curr. Org. Chem. 2009, 13, 1432. (g) Yu, X.; Wang, W. Org. Biomol. Chem. 2008, 6, 2037. (h) Enders, D.; Grondal, C.; Hüttl, M. R. M. Angew. Chem. Int. Ed. 2007, 46, 1570.

(2) For selected general reviews on hydrogen bonding, see: (a) Lu, T.; Wheeler, S. E. Chem. Eur. J. 2013, 19, 15141. (b) Wurm, F. R.; Klok, H.-A. Chem. Soc. Rev. 2013, 42, 8220. (c) Brière, J.-F.; Oudeyer, S.; Dalla, V.; Levacher, V. Chem. Soc. Rev. 2012, 41, 1696. (d) Alemán, J.; Parra, A.; Jiang, H.; Jørgensen, K. A. Chem. Eur. J. 2011, 17, 6890. (e) Knowles, R. R.; Jacobsen, E. N. Proc. Natl. Acad. Sci. U.S.A. 2010, 107, 20678. (f) Etzenbach-Effers, K.; Berkessel, A. Top. Curr. Chem. 2009, 291, 1. (g) Takemoto, Y. Chem. Pharm. Bull. 2010, 58, 593. (h) Kotke, M.; Schreiner, P. R. In Hydrogen Bonding in Organic Synthesis; Pihko, P. M., Ed.; Wiley-VCH: Weinheim, 2009, 141. (i) Zhang, Z.; Schreiner, P. R. Chem. Soc. Rev. 2009, 38, 1187. (j) Doyle, A. G.; Jacobsen, E. N. Chem. Rev. 2007, 107, 5713. (k) Taylor, M. S.; Jacobsen, E. N. Angew. Chem. Int. Ed. 2006, 45, 1520. (1) Schreiner, P. R. Chem. Soc. Rev. 2003, 32, 289.

(3) For reviews on tetrahydropyran syntheses, see: (a) Olier, C.; Kaafarani, M.; Gastaldi, S.; Bertrand, M. P. Tetrahedron 2010, 66, 413. (b) Larrosa, I.; Romea, P.; Urpí, F. Tetrahedron 2008, 64, 2683. (c) Clarke, P. A.; Santos, S. Eur. J. Org. Chem. 2006, 2045. (d) Shindo, M. Top. Heterocycl. Chem. 2006, 5, 179. (e) Lee, E. Pure Appl. Chem. 2005, 77, 2073. (f) Yeung, K.-S.; Paterson, I. Chem. Rev. 2005, 105, 4237. (g) Boivin, T. L. B. Tetrahedron 1987, 43, 3309.

(4) For selected examples of tetrahydropyran syntheses, see: (a) Dange, N. S.; Hong, B-C.; Lan, D-J.; Liao, J-H.; Lee, G.H. Eur. J. Org. Chem. 2013, 12, 2472. (b) Wang, Y.; Zhu, S.;
Ma, D. Org. Lett. 2011, 13, 1602. (c) Lee, K.; Kim, H.; Hong, J. Eur. J. Org. Chem. 2012, 1025. (d) Ishikawa, H.; Sawano, S.; Yasui, Y.; Shibata, Y.; Hayashi, Y. Angew. Chem. Int. Ed. 2011, 50, 3774. (e) Liu, L.; Floreancig, P. E. Angew. Chem. Int. Ed. 2010, 49, 3069. (f) Fuwa, H.; Noto, K.; Sasaki, M. Org. Lett. 2010, 12, 1636. (g) Trost, B. M.; Gutierrez, A. C.; Livingston, R. C. Org. Lett. 2009, 11, 2539. (h) Chandrasekhar, S.; Mallikarjun, K.; Pavankumarreddy, G.; Veeramohana, Rao. K.; Jagadeesh, B. Chem. Commun. 2009, 4985. (i) Cao, C-L.; Zhou, Y-Y.; Sun, X-L.; Tang, Y.; Li, Y-X.; Li, G-Y.; Sun, J. Chem. Eur. J. 2009, 15, 11384. (j) Gotoh, H.; Okamura, D.; Ishikawa, H.; Hayashi, Y. Org. Lett. 2009, 11, 4056. (k) De Brab, J. K.; Liu, B.; Qian, M. Org. Lett. 2008, 10, 2533. (1) Hiebel, M.-A.; Pelotier, B.; Goekjian, P.; Piva, O. Eur. J. Org. Chem. 2008, 713.

(5) (a) Enders, D.; Urbanietz, G.; Raabe, G. Synthesis 2011, 1905. (b) Enders, D.; Urbanietz, G.; Hahn, R.; Raabe, G. Synthesis 2012, 44, 773. (c) Ramachary, D. B.; Madhavachary, R.; Prasad, M. S. Org. Biomol. Chem. 2012, 10,5825 .

(6) For some selected examples of (E)-2-nitro-3-phenylprop-2en-1-ol in synthesis, see: (a) Beck, A. K.; Duschmale, J.; Ebert, M-O.; Purkayastha, N.; Schweizer, W. B.; Seebach, D.; Sun, X.; Wennemers, H.; Benohoud, M.; Hayashi, Y.; Mukaiyama, T.; Reiher, M. Helv. Chim. Acta 2013, 96, 799. (b) An, J.; Lu, L-Q.; Wang, T.; Xiao, W-J.; Yang, Q-Q. Org. Lett. 2013, 15, 542. (c) Talavera, G.; Reyes, E.; Vicario, J. L.; Carrillo, L. Angew. Chem. Int. Ed. 2012, 51, 4104. (d) Parra, A.; Reboredo, S.; Alemán, J. Angew. Chem. Int. Ed. 2012, 51, 9734. (e) Fioravanti, S.; Pellacani, L.; Vergari, M. C. Org. Biomol. Chem. 2012, 10, 524. (f) Guo, Z-W.; Xie, J-W.; Chen, C.; Zhu, W-D. Org. Biomol. Chem. 2012, 10, 8471. (g) Rai, A.; Yadav, L. D. S. Org. Biomol. Chem. 2011, 9, 8058. (h) Bakthadoss, M.; Sivakumar, N. Synlett 2011, 1296. (i) Bakthadoss, M.; Shivakumar, N.; Deraraj, A. Synthesis 2011, 611.

(7) The (E)-3-aryl-2-nitroprop-2-en-1-ols were prepared from the corresponding $(E)$ - $\beta$-nitrostyrenes and formaldehyde in THF according to a literature procedure: see ref. $6 \mathrm{i}$.

(8) Malerich, J. P.; Hagihara, K.; Rawal, V. H. J. Am. Chem. Soc. 2008, 130, 14416.

(9) Tripathi, C. B.; Mukherjee, S. Angew. Chem. Int. Ed. 2013, 52,8450

(10) CCDC 965195 (trans-3g) contains the supplementary crystallographic data for this paper. These data can be obtained free of charge from The Cambridge Crystallographic Data Centre via www.ccdc.cam.ac.uk/data_request/cif or by writing to the Cambridge Crystallographic Data Centre, 12, Union Road, Cambridge CB2 1EZ, UK; fax: +44(1223)336033; E-mail: deposit@ccdc.cam.ac.uk. 